\title{
A hot Jupiter spectral sequence with evidence for compositional diversity
}

\author{
Megan Mansfield ( $\nabla$ meganmansfield@uchicago.edu ) \\ University of Chicago https://orcid.org/0000-0003-4241-7413 \\ Michael Line \\ Arizona State University \\ Jacob Bean \\ University of Chicago \\ Jonathan Fortney \\ University of California, Santa Cruz \\ Vivien Parmentier \\ University of Oxford \\ Eliza Kempton \\ University of Maryland https://orcid.org/0000-0002-1337-9051 \\ David Sing \\ Johns Hopkins University https://orcid.org/0000-0001-6050-7645 \\ Mercedes Lopez-Morales \\ Harvard University
}

Claire Baxter

University of Amsterdam

Jean-Michel Désert

University of Amsterdam

Mark Swain

Jet Propulsion Laboratory

Gael Roudier

NASA Jet Propulsion Laboratory

\section{Research Article}

Keywords: hot Jupiters, thermal emission spectra, spectral sequence, compositional diversity

Posted Date: October 26th, 2020

DOI: https://doi.org/10.21203/rs.3.rs-93825/v1 
License: (c) (i) This work is licensed under a Creative Commons Attribution 4.0 International License. Read Full License

Version of Record: A version of this preprint was published at Nature Astronomy on October 21st, 2021. See the published version at https://doi.org/10.1038/s41550-021-01455-4. 


\title{
A hot Jupiter spectral sequence with evidence for compositional diversity
}

\author{
Megan Mansfield ${ }^{1, *}$, Michael R. Line ${ }^{2}$, Jacob L. Bean ${ }^{3}$, Jonathan J. Fortney ${ }^{4}$, Vivien \\ ${ }_{4}$ Parmentier $^{5}$, Eliza M.-R. Kempton ${ }^{6}$, David K. Sing ${ }^{7}$, Mercedes López-Morales ${ }^{8}$, Claire \\ ${ }_{5}$ Baxter $^{9}$, Jean-Michel Désert ${ }^{9}$, Mark R. Swain ${ }^{10}$, and Gael M. Roudier ${ }^{10}$ \\ $6{ }^{1}$ Department of Geophysical Sciences, University of Chicago, 5734 S. Ellis Avenue, Chicago, IL 60637, USA \\ $7{ }^{2}$ School of Earth and Space Exploration, Arizona State University, Tempe, AZ 85281, USA \\ $8{ }^{3}$ Department of Astronomy \& Astrophysics, University of Chicago, Chicago, IL 60637, USA \\ $9{ }^{4}$ Department of Astronomy and Astrophysics, University of California, Santa Cruz, CA 95064, USA \\ ${ }^{5}$ Department of Physics, University of Oxford, Oxford, OX1 3PU, UK \\ $1{ }^{6}$ Department of Astronomy, University of Maryland, College Park, MD 20742, USA \\ ${ }^{7}$ Department of Physics \& Astronomy, Johns Hopkins University, Baltimore, MD 21218, USA \\ ${ }_{3}^{8}$ Center for Astrophysics | Harvard \& Smithsonian, Cambridge, MA 01238, USA \\ ${ }_{14}^{9}$ Anton Pannekoek Institute for Astronomy, University of Amsterdam, $1098 \mathrm{XH}$ Amsterdam, The Netherlands \\ $5{ }^{10}$ NASA Jet Propulsion Laboratory, California Institute of Technology, Pasadena, CA 91109, USA \\ 6 *Corresponding author: meganmansfield@uchicago.edu
}

\section{ABSTRACT}

The emergent spectra of close-in, giant exoplanets ("hot Jupiters") are believed to be distinct from those of young gas giants and brown dwarfs with similar effective temperatures because these objects are primarily heated from above by their host stars rather than internally from the release of energy from their formation ${ }^{1}$. Theoretical models predict a continuum of dayside spectra for hot Jupiters as a function of irradiation level, with the coolest planets having absorption features in their spectra, intermediate-temperature planets having emission features due to thermal inversions, and the hottest planets having blackbody-like spectra due to molecular dissociation and continuum opacity from the $\mathrm{H}^{-}$ion ${ }^{2-4}$. Absorption and emission 18 features have been detected in the spectra of a number of individual hot Jupiters ${ }^{5,6}$, and population-level trends have been observed in photometric measurements ${ }^{7-11}$. However, there has been no unified, population-level study of the thermal emission spectra of hot Jupiters such as has been done for brown $\mathrm{dwarfs}^{12}$ and transmission spectra of hot Jupiters ${ }^{13}$. Here we show that hot Jupiter secondary eclipse spectra centered around a water absorption band at $1.4 \mu \mathrm{m}$ follow a common trend in water feature strength with temperature. The observed trend is broadly consistent with the predictions of self-consistent one-dimensional models for how the thermal structures of solar composition planets vary with irradiation level. Nevertheless, the ensemble of planets exhibits significant scatter around the mean trend. The spread can be accounted for if the planets have modest variations in metallicity and/or elemental abundance ratios, which is expected from planet formation models ${ }^{14-17}$.

We performed a statistical analysis of 14 hot Jupiter sec- 34 in spatial scanning mode in this wavelength region. Suppleondary eclipse spectra obtained with the Wide Field Camera $3{ }_{35}$ mentary Table 1 contains detailed information on each of the (WFC3) instrument on the Hubble Space Telescope (HST) us- 36 eight literature results we considered. We limited this study to ing the G141 grism between 1.1 and $1.7 \mu \mathrm{m}$. This bandpass ${ }_{37}$ observations made in the spatial scanning mode and excluded is primarily sensitive to water vapor in exoplanet atmospheres, зв stare mode observations because previous work has shown and the largest molecular feature in this wavelength range 39 that instrument systematics are better understood in scanning is a water vapor absorption band centered at about $1.4 \mu \mathrm{m}$. 40 mode, so the data derived from scanning mode observations Over the last decade a large sample of exoplanets have been 41 are less likely to be complicated by inaccurately corrected observed using WFC3+G141 to understand their atmospheric 42 systematics ${ }^{19}$. The planets in this study have observed daywater abundances ${ }^{13,18}$, and it has become an important tool in ${ }_{43}$ side temperatures in the HST/WFC3+G141 bandpass between understanding exoplanet atmospheres.

We analyzed six new data sets following the data reduction procedure outlined in the Methods. We combined these six 46 new analyses with eight results from the literature to form a 47 the thermal emission spectra of a subset of planets observed complete sample of planets observed with $H S T / \mathrm{WFC} 3+\mathrm{G} 14148$ with $H S T$. This study expands on that work by uniformly an- 
49 alyzing all $H S T$ thermal emission spectra and performing a 52 50 more comprehensive analysis of the changes in their spectra 5 51 with temperature.

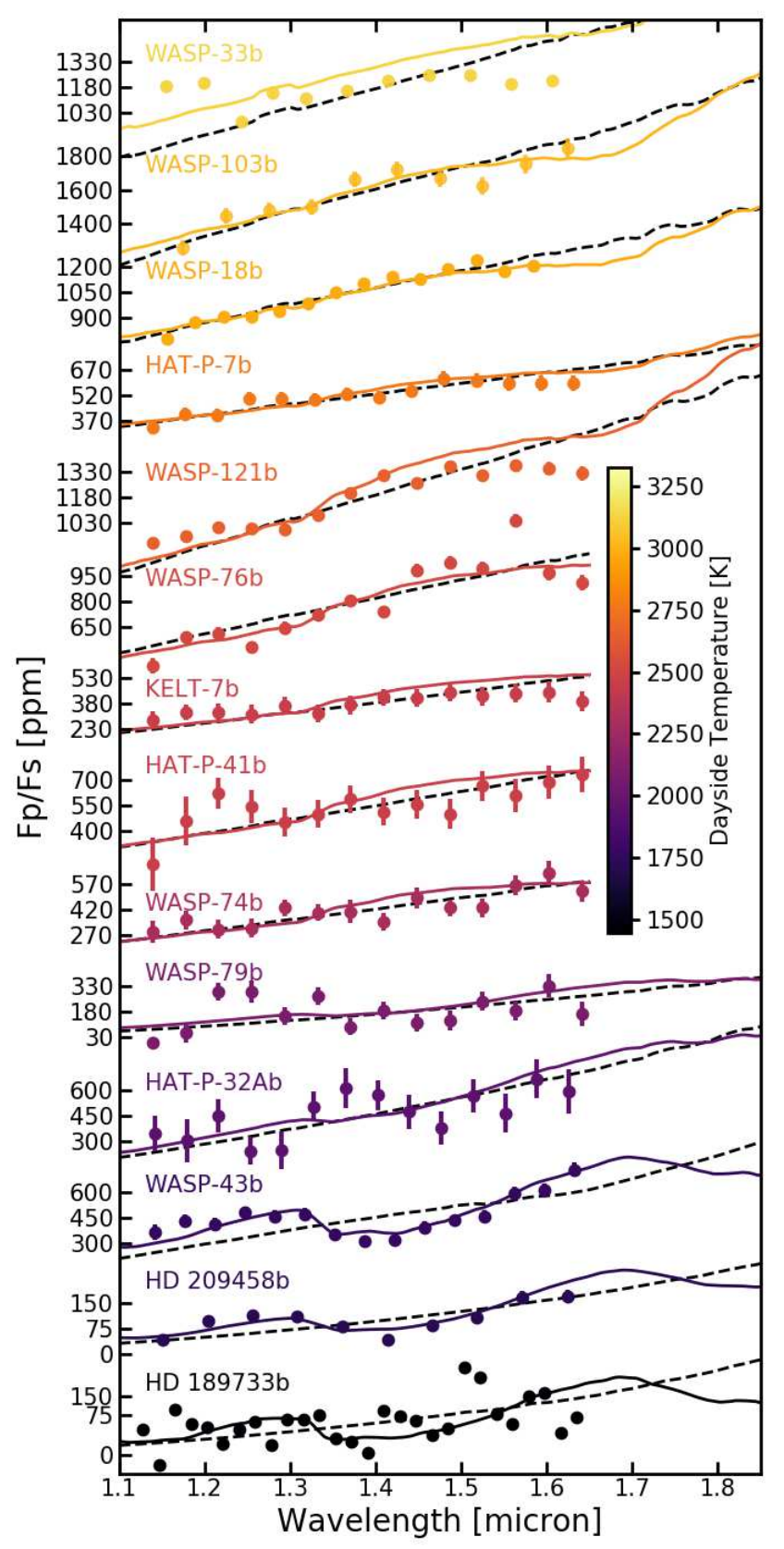

Figure 1. Secondary eclipse spectra for all 14 hot Jupiters considered in this study. Data sets are colored by dayside temperature, which is measured as described in the Methods and shown by the colorbar. Solid lines indicate interpolations from our solar composition fiducial model grid (see the Methods section for a description), while dashed lines indicate best-fit blackbodies. Note that for several data sets, the error bars are smaller than the point size.
We created a new grid of cloud-free irradiated 1D self3 consistent radiative-convective-thermochemical equilibrium 54 models to compare to the dayside HST/WFC3+G141 thermal 55 emission observations. These models were created using the 56 Sc-CHIMERA framework ${ }^{4,9,20-23}$ which includes a broad ar57 ray of opacity sources that are important for the temperature 58 range explored here, including atomic and ionic opacities that 59 are relevant at the high temperatures of ultra-hot Jupiters ${ }^{24}$. A 60 full description of the models and complete list of opacities 61 can be found in the Methods.

62 Figure 2 shows the temperature-pressure profiles and re63 sultant secondary eclipse spectra for our fiducial model, which 64 uses system parameters for a standard hot Jupiter (stellar effec65 tive temperature $T_{\text {eff }}=5300 \mathrm{~K}$, planetary gravity $g=10 \mathrm{~m} / \mathrm{s}^{2}$, 66 planetary metallicity $\left[\frac{\mathrm{M}}{\mathrm{H}}\right]=0.0$, planetary carbon-to-oxygen 67 abundance ratio $\frac{\mathrm{C}}{\mathrm{O}}=0.55$, and planetary internal temperature $\left.{ }_{68} T_{\text {int }}=150 \mathrm{~K}\right)$. Models at different temperatures were created 69 by scaling the incident stellar flux to match the specified ir70 radiation temperature. Figure 2 also shows the ratio of the 71 absorption mean opacity $\left(\kappa_{J}\right)$ to the Planck mean opacity $72\left(\kappa_{B}\right)$ as a function of equilibrium temperature at a pressure of $7310^{-2}$ bar, which is approximately the photospheric pressure 74 in the HST/WFC3 bandpass (see the Methods for a full de75 scription of these opacities). This ratio describes the relative 76 efficiency of stellar absorption vs. thermal re-radiation at that 77 layer in the planet's atmosphere ${ }^{25}$.

In addition to the fiducial model grid, we created models 79 with a variety of atmospheric/system parameters to see how 80 individual parameters impact the $1 \mathrm{D}$ vertical structure and 81 resulting population level trends observed in the dayside emis82 sion spectra. We examined models with stellar $T_{\text {eff }}=3300 \mathrm{~K}$, $834300 \mathrm{~K}, 6300 \mathrm{~K}, 7200 \mathrm{~K}$, and $8200 \mathrm{~K}$; planetary gravity, ${ }_{84} g=1 \mathrm{~m} / \mathrm{s}^{2}$ and $100 \mathrm{~m} / \mathrm{s}^{2}$; metallicity, $\left[\frac{\mathrm{M}}{\mathrm{H}}\right]=-1.5$ and 1.5 ; 85 and $\frac{\mathrm{C}}{\mathrm{O}}=0.01$ and 0.85 . We also included a model where 86 the internal temperature varies with the planetary irradiation 87 temperature to capture the internal entropy change that could 88 be the cause of the hot Jupiter radii inflation ${ }^{26}$. Furthermore, 89 we tested models in which the TiO and VO opacity were re90 moved ad hoc until temperatures above $2000 \mathrm{~K}, 2500 \mathrm{~K}$, or $13000 \mathrm{~K}$ in order to simulate cold-trapping in cooler regions 92 of the atmosphere ${ }^{3,27,28}$ (see the Methods section for a full 93 description of these models). For all of these models, only 94 one parameter was varied at a time while the other parameters 95 were held fixed to the values in the fiducial model (e.g., a slice 96 along a given parameter dimension). 

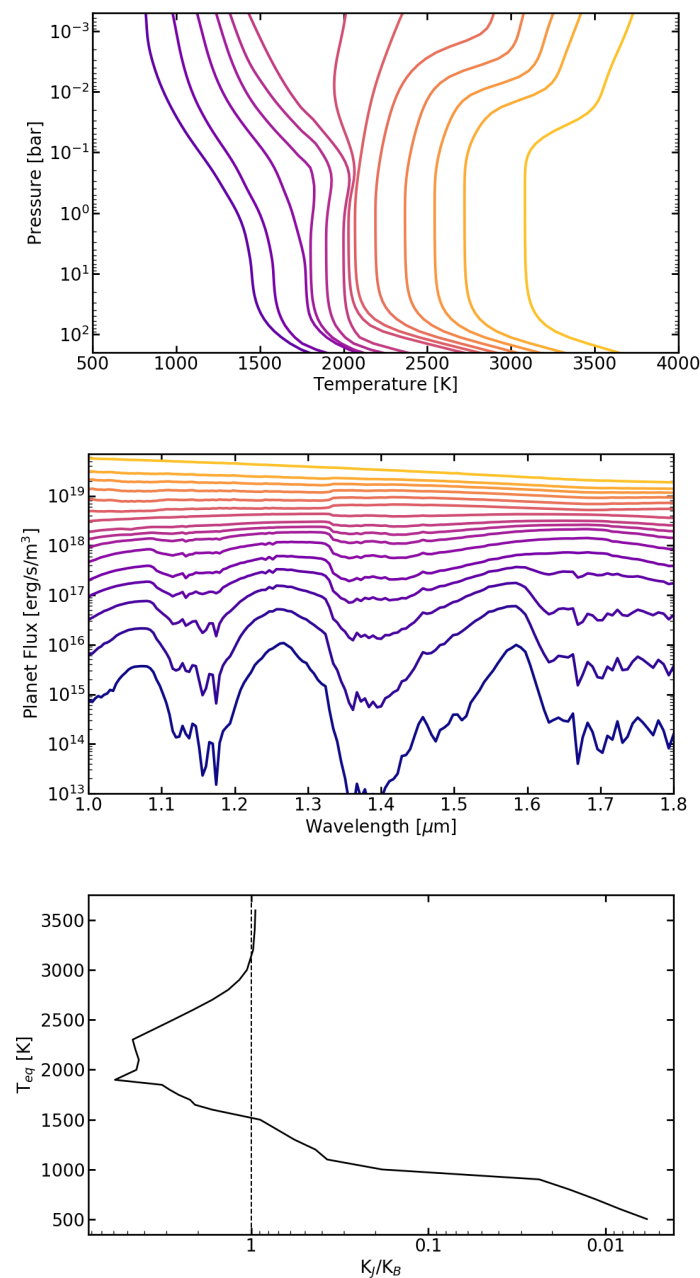

Figure 2. Temperature-pressure (T-P) profiles (top) and resulting dayside planet fluxes (middle) for the fiducial model grid, which covers approximately the same range of temperatures as spanned by the observations. The full model specifications are detailed in the Methods. The fiducial model uses a $5300 \mathrm{~K}$ stellar effective temperature, a solar composition planetary atmosphere $\left(\left[\frac{\mathrm{M}}{\mathrm{H}}\right]=0.0\right.$ and $\left.\frac{\mathrm{C}}{\mathrm{O}}=0.55\right)$, a planetary gravity of $10 \mathrm{~m} / \mathrm{s}^{2}$, and a planet internal temperature of $150 \mathrm{~K}$. Blue and yellow lines show models with the coolest and warmest irradiation temperatures, respectively. For clarity, only every other model in the grid is shown here. The bottom panel shows the ratio of the absorption mean opacity $\left(\kappa_{J}\right)$ to the Planck mean opacity $\left(\kappa_{B}\right)$ as a function of equilibrium temperature in these models at a pressure of $10^{-2}$ bar. This ratio describes the relative efficiency of heating vs. cooling in the models ${ }^{25}$, and a ratio of $\kappa_{J} / \kappa_{B}>1$ generally indicates the presence of a thermal inversion in the temperature-pressure profile. This panel is plotted with temperature on the y-axis for ease of comparison to Figure 3. Our models predict three primary spectral regimes. At ${ }_{148}$ abundance ratios that fall within the range of commonly ex-
98 the lowest dayside temperatures $\left(T_{\text {day }}<2100 \mathrm{~K}\right.$ for the fidu99 cial model), the models exhibit absorption features due to 100 monotonically decreasing temperature profiles. At interme101 diate temperatures $\left(2100 \mathrm{~K}<T_{\text {day }}<3000 \mathrm{~K}\right.$ for the fiducial 102 model), the modeled thermal structures exhibit a rising tem${ }_{103}$ perature with increasing altitude (decreasing pressure) due to 104 the gas-phase onset of $\mathrm{TiO}$ and $\mathrm{VO}$ which push $K_{J} / K_{B}>1$, in 105 turn causing emission features. At the highest temperatures ${ }_{106}\left(T_{\text {day }}>3000 \mathrm{~K}\right.$ for the fiducial model $)$, the models still show 107 strong thermal inversions (becoming stronger primarily due to 108 the dissociation of water, an efficient coolant) but the resulting 109 secondary eclipse spectra are relatively featureless because of 110 a combination of high-temperature effects such as molecular 111 dissociation and the onset of $\mathrm{H}^{-}$opacity, which cause all the $112 \mathrm{WFC} 3+\mathrm{G} 141$ wavelengths to probe the same altitude/pressure 113 level, hence brightness temperature ${ }^{3,4,20,21,24}$. The exact tem114 peratures of the transitions between these regimes, as well as 115 the strength of absorption and emission features present in the 116 models, depend on the parameters of each set of models.

117 For both the models and the population of 14 observed hot ${ }_{118}$ Jupiters, we examined the degree of absorption or emission 119 observed in the water feature at $1.4 \mu \mathrm{m}$, the primary feature 120 in the $H S T / \mathrm{WFC} 3+\mathrm{G} 141$ bandpass. We quantified their devi121 ation from a blackbody using an $H S T$ water feature strength 122 metric, which is illustrated in Supplementary Figure 1. For ${ }_{123}$ each data set, we first fit a blackbody to the two "out-of124 band" regions of the spectrum, which have wavelengths of ${ }_{125} 1.22-1.33 \mu \mathrm{m}$ and $1.53-1.61 \mu \mathrm{m}$ and are defined based 126 on where the models in Figure 2 show minimal water opacity. ${ }_{127}$ The temperature of this blackbody is referred to throughout ${ }_{128}$ this paper as the observed dayside temperature $\left(T_{\text {day }}\right)$ in this 129 bandpass. The water feature strength is then defined as

$$
S_{H_{2} O}=\log _{10}\left(\frac{F_{B, i n}}{F_{o b s, i n}}\right),
$$

${ }^{130}$ where $F_{B, i n}$ and $F_{o b s, i n}$ are the flux of the fitted blackbody ${ }_{131}$ and the observed data, respectively, in the "in-band" region ${ }_{132}$ shown in Supplementary Figure 1. The "in-band" wavelength ${ }_{133}$ region extends from $1.35-1.48 \mu \mathrm{m}$ and captures the center 134 of the primary water band observed in the HST/WFC3 band135 pass. Supplementary Figure 2 shows all 14 secondary eclipse ${ }_{36}$ spectra with the "out-of-band" and "in-band" regions shaded. ${ }_{37}$ From this definition, $\mathrm{S}_{\mathrm{H}_{2} \mathrm{O}}$ will have a positive value when 138 a water feature is observed in absorption, a negative value when a feature is observed in emission, and a value of zero if a blackbody is observed.

${ }_{141} \quad$ Figures 3 and 4 show the observed $H S T$ water feature 12 strengths for the sample of 14 hot Jupiter emission spectra 143 compared to those of the models. Figure 3 shows that the 144 observed HST/WFC3 feature strengths generally fall within ${ }_{45}$ the region of parameter space spanned by the models, with ${ }_{46}$ almost all of the planets fully within the predicted spread of 147 the models. The models considered here assume elemental 
149 pected outcomes from planet formation models ${ }^{14-17}$. We find 173 ratio had a significant impact on the predicted $H S T /$ WFC3 150 that varying parameters in these simple models can explain the 174 water feature strengths. We found the observed scatter could 151 observed hot Jupiter population without having to appeal to 175 be explained if the planets have atmospheric metallicites be152 less likely outcomes of planet formation (e.g., $\mathrm{C} / \mathrm{O}>1^{14,15,17}$ ) 176 tween $0.03-30 \mathrm{x}$ solar and $\mathrm{C} / \mathrm{O}$ ratios between $0.01-0.85$ 153 or exotic chemistry.

$177(0.02-1.5 x$ solar $)$. Such variation is expected from planet

Although the observed population of hot Jupiter emission 178 formation models ${ }^{14,15}$ and has been suggested by a handful of 5 spectra generally matches our model predictions, we find that 179 transmission spectra studies (e.g., $\left.{ }^{17}\right)$. The scatter we observed 156 no single model track can match all of the observations at bet-180 in emission spectra lends further support to the concept of

\section{References}

1. Showman, A. P., Tan, X. \& Parmentier, V. Atmospheric Dynamics of Hot Giant Planets and Brown Dwarfs. arXiv e-prints arXiv:2007.15363 (2020). 2007.15363.

2. Fortney, J. J., Lodders, K., Marley, M. S. \& Freedman, R. S. A Unified Theory for the Atmospheres of the Hot and Very Hot Jupiters: Two Classes of Irradiated Atmospheres. ApJ 678, 1419-1435 (2008). DOI 10.1086/528370. 0710.2558.

3. Parmentier, V. et al. From thermal dissociation to condensation in the atmospheres of ultra hot Jupiters: WASP-121b in context. A\&A 617, A110 (2018). DOI 10.1051/0004-6361/201833059. 1805.00096.

4. Arcangeli, J. et al. $\mathrm{H}^{-}$Opacity and Water Dissociation in the Dayside Atmosphere of the Very Hot Gas Giant WASP-18b. ApJL 855, L30 (2018). DOI 10.3847/2041-8213/aab272. 1801.02489.

5. Kreidberg, L. et al. A Precise Water Abundance Measurement for the Hot Jupiter WASP-43b. ApJL 793, L27 (2014). DOI 10.1088/2041-8205/793/2/L27. 1410.2255.

6. Mikal-Evans, T. et al. Confirmation of water emission in the dayside spectrum of the ultrahot Jupiter WASP-121b. MNRAS 496, 1638-1644 (2020). DOI 10.1093/mnras/staa1628. 2005.09631.

7. Keating, D., Cowan, N. B. \& Dang, L. Uniformly hot nightside temperatures on short-period gas giants. Nat. Astron. 3, 1092-1098 (2019). DOI 10.1038/s41550-019-0859-z. 1809.00002.

8. Beatty, T. G. et al. Spitzer Phase Curves of KELT-1b and the Signatures of Nightside Clouds in Thermal Phase Observations. AJ 158, 166 (2019). DOI 10.3847/1538-3881/ab33fc. 1808.09575.

9. Baxter, C. et al. A transition between the hot and the ultra-hot Jupiter atmospheres. A\&A 639, A36 (2020). DOI 10.1051/0004-6361/201937394. 2007.15287.

10. Garhart, E. et al. Statistical Characterization of Hot Jupiter Atmospheres Using Spitzer's Secondary Eclipses. AJ 159, 137 (2020). DOI 10.3847/1538-3881/ab6cff. 1901.07040.

11. Dransfield, G. \& Triaud, A. H. M. J. Colour-magnitude diagrams of transiting Exoplanets - III. A public code, nine strange planets, and the role of Phosphine. arXiv e-prints arXiv:2008.00995 (2020). 2008.00995.

12. Manjavacas, E. et al. Cloud Atlas: Hubble Space Telescope Near-infrared Spectral Library of Brown Dwarfs, Planetarymass Companions, and Hot Jupiters. AJ 157, 101 (2019). DOI 10.3847/1538-3881/aaf88f. 1812.03963. 


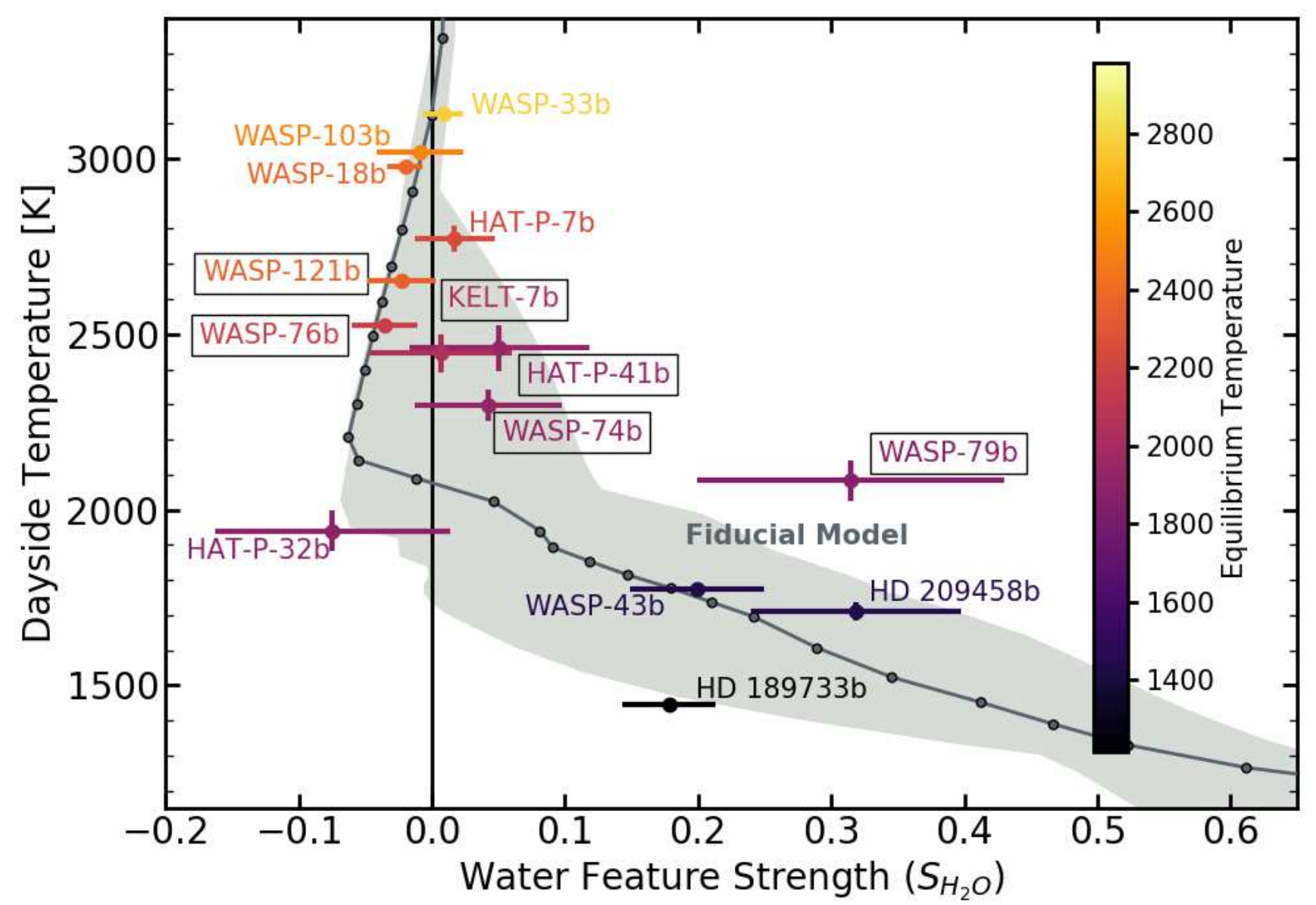

Figure 3. HST water feature strength diagram comparing observed secondary eclipse spectra to the model predictions in Figure 2. The y-axis shows the temperature of a blackbody fit to the "out-of-band" regions defined in Supplementary Figure 1, which is the observed dayside temperature $T_{\text {day }}$. The x-axis shows the strength of the observed feature in the water band at $1.4 \mu \mathrm{m}$ compared to this blackbody, as defined by Equation 1. Featureless, blackbody-like spectra have $S_{\mathrm{H}_{2} \mathrm{O}}=0$ and absorption/emission features have positive/negative colors, respectively. The gray line and points show the fiducial models pictured in Figure 2. The light gray shaded region shows the full range of model predictions assuming different values for the stellar effective temperature; the temperature where $\mathrm{TiO}$ opacity becomes important; and the planet gravity, $\mathrm{C} / \mathrm{O}$ ratio, metallicity, and internal heat. Colored points with error bars show all planets with $H S T / \mathrm{WFC} 3$ spectra observed in the spatial scanning mode, and boxes around planet names indicate new data reductions in this publication. The color scale indicates the planetary equilibrium temperature. The error bars include uncertainties in the stellar effective temperature. 

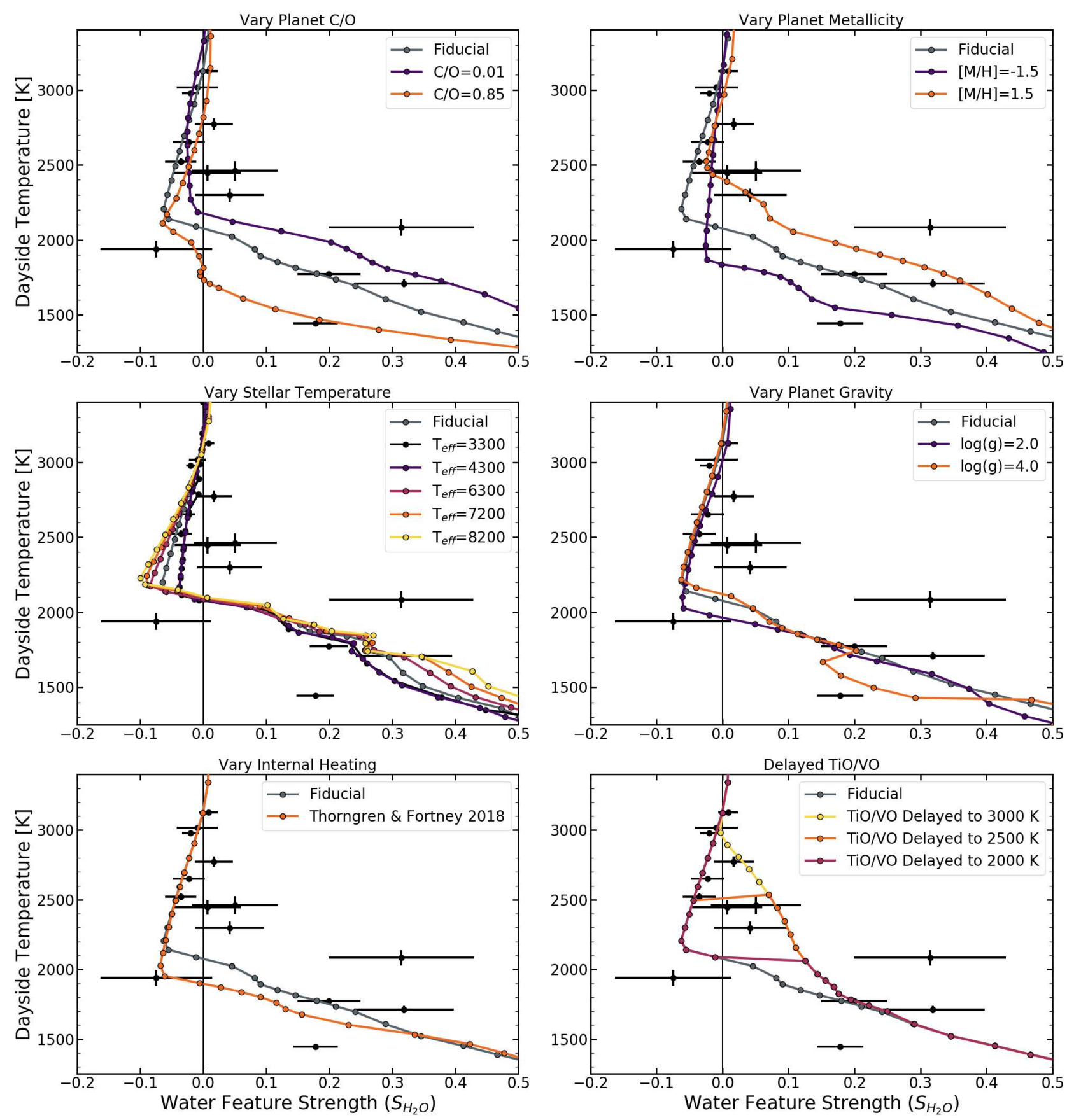

Figure 4. Diagrams illustrating the change in HST water feature strength from models with different parameters. All diagrams show the observed data as black points with error bars, while the lines show tracks for models with varying C/O ratio (top left), metallicity (top right), stellar temperature (middle left), gravity (middle right), internal heating (bottom left), and the temperature to which $\mathrm{TiO}$ opacity was ignored (bottom right). In each case all other parameters are held fixed at the fiducial model values. The error bars include uncertainties in the stellar effective temperature. We found that changing the stellar temperature, planetary gravity, and internal heating in our models had little impact on the derived water feature strengths, and changing the $\mathrm{TiO} / \mathrm{VO}$ only had an impact at intermediate temperatures, but changing the atmospheric $\mathrm{C} / \mathrm{O}$ ratio and metallicity can explain the diversity of observed secondary eclipse spectra. 
263 planets. At the time this study was begun, these were all of 272

${ }_{264}$ the remaining secondary eclipse data sets in the $H S T$ archive 273

265

266

267

$$
268
$$
59-62 (2016). DOI 10.1038/nature16068. 1512.04341. (2017). DOI 10.1093/mnras/stx260. 1611.03128. 1910.13171. 1704.05413. 1406.7567. 10.3847/1538-3881/aac497. 1805.00424. (2019). DOI 10.3847/1538-4357/ab1485. 1903.12183. 637X/817/1/17. 1511.05528.

14. Mordasini, C., van Boekel, R., Mollière, P., Henning, T. \& Benneke, B. The Imprint of Exoplanet Formation History on Observable Present-day Spectra of Hot Jupiters. ApJ 832, 41 (2016). DOI 10.3847/0004-637X/832/1/41. 1609. 03019.

15. Ali-Dib, M. Disentangling hot Jupiters formation location from their chemical composition. MNRAS 467, 2845-2854

16. Madhusudhan, N., Bitsch, B., Johansen, A. \& Eriksson, L. Atmospheric signatures of giant exoplanet formation by pebble accretion. MNRAS 469, 4102-4115 (2017). DOI 10.1093/mnras/stx1139. 1611.03083.

17. Cridland, A. J., van Dishoeck, E. F., Alessi, M. \& Pudritz, R. E. Connecting planet formation and astrochemistry. A main sequence for $\mathrm{C} / \mathrm{O}$ in hot exoplanetary atmospheres. A\&A 632, A63 (2019). DOI 10.1051/0004-6361/201936105.

18. Tsiaras, A. et al. A Population Study of Gaseous Exoplanets. AJ 155, 156 (2018). DOI 10.3847/1538-3881/aaaf75.

19. Stevenson, K. B., Bean, J. L., Madhusudhan, N. \& Harrington, J. Deciphering the Atmospheric Composition of WASP12b: A Comprehensive Analysis of its Dayside Emission. ApJ 791, 36 (2014). DOI 10.1088/0004-637X/791/1/36.

20. Kreidberg, L. et al. Global Climate and Atmospheric Composition of the Ultra-hot Jupiter WASP-103b from HST and Spitzer Phase Curve Observations. AJ 156, 17 (2018). DOI 10.3847/1538-3881/aac3df. 1805.00029.

21. Mansfield, M. et al. An HST/WFC3 Thermal Emission Spectrum of the Hot Jupiter HAT-P-7b. AJ 156, 10 (2018). DOI

22. Piskorz, D. et al. Ground- and Space-based Detection of the Thermal Emission Spectrum of the Transiting Hot Jupiter KELT-2Ab. AJ 156, 133 (2018). DOI 10.3847/1538-3881/aad781. 1809.05615.

23. Zalesky, J. A., Line, M. R., Schneider, A. C. \& Patience, J. A Uniform Retrieval Analysis of Ultra-cool Dwarfs. III. Properties of Y Dwarfs. ApJ 877, 24 (2019). DOI 10.3847/1538-4357/ab16db. 1903.11658.

24. Lothringer, J. D., Barman, T. \& Koskinen, T. Extremely Irradiated Hot Jupiters: Non-oxide Inversions, $\mathrm{H}^{-}$Opacity, and Thermal Dissociation of Molecules. ApJ 866, 27 (2018). DOI 10.3847/1538-4357/aadd9e. 1805.00038.

25. Lothringer, J. D. \& Barman, T. The Influence of Host Star Spectral Type on Ultra-hot Jupiter Atmospheres. ApJ 876, 69

26. Thorngren, D., Gao, P. \& Fortney, J. J. The Intrinsic Temperature and Radiative-Convective Boundary Depth in the Atmospheres of Hot Jupiters. ApJL 884, L6 (2019). DOI 10.3847/2041-8213/ab43d0. 1907 . 07777.

27. Parmentier, V., Showman, A. P. \& Lian, Y. 3D mixing in hot Jupiters atmospheres. I. Application to the day/night cold trap in HD 209458b. A\&A 558, A91 (2013). DOI 10.1051/0004-6361/201321132. 1301.4522.

28. Beatty, T. G. et al. Evidence for Atmospheric Cold-trap Processes in the Noninverted Emission Spectrum of Kepler-13Ab Using HST/WFC3. AJ 154, 158 (2017). DOI 10.3847/1538-3881/aa899b. 1612.06409.

29. Greene, T. P. et al. Characterizing Transiting Exoplanet Atmospheres with JWST. ApJ 817, 17 (2016). DOI 10.3847/0004-

30. Snellen, I. A. G., de Kok, R. J., de Mooij, E. J. W. \& Albrecht, S. The orbital motion, absolute mass and high-altitude winds of exoplanet HD209458b. Nat. 465, 1049-1051 (2010). DOI 10.1038/nature09111. 1006.4364.

\section{Methods}

\section{New Observations and Data Reduction} that had not been published yet. Since we began this project,274 extraction procedure ${ }^{35}$ and masked cosmic rays. To subtract results for three planets have been published ${ }^{6,31-33}$. In all of 275 the background out of each frame, we visually inspected the these cases, our reductions produced spectra consistent with 276 images to find a clear background spot on the detector and the published results. Supplementary Table 2 lists the details 277 subtracted the median of this background area. The uncerWe reduced the data using the data reduction pipeline
described in Kreidberg et al. $(2014)^{34}$. We used an optimal

269 of these observations, which included single eclipses of HAT270 P-41b, KELT-7b, WASP-74b, WASP-76b, and WASP-79b, as 271 well as five eclipses of WASP-121b. We reduced the data using the data reduction pipeline 
curves with a model in the form
fit both the white light curvc

$$
M(t)=E(t)\left(c s+v t_{v i s}\right)\left(1-e^{-r_{1} t_{o r b}-r_{2}}\right),
$$

289 where $\mathrm{M}(\mathrm{t})$ is the modeled flux, $\mathrm{E}(\mathrm{t})$ is an eclipse model found 290 using batman ${ }^{36}$, and the rest of the equation is a systematics 291 model based on Berta et al. (2012) 37 . In this systematics 292 model, $c$ is a normalization constant, $s$ is a scaling factor to 293 account for an offset in normalization between scan direc294 tions, $v$ is a visit-long linear trend, $t_{v i s}$ is the time since the 295 beginning of the visit, $r_{1}$ and $r_{2}$ are the amplitude and time 296 constant of an orbit-long exponential ramp, respectively, and $297 t_{\text {orb }}$ is the time since the beginning of the orbit. For both the

free parameter in the eclipse model was the planet-to-star flux ratio $F_{p} / F_{s}$.

The single eclipses observed for most of these planets had poor coverage of ingress and egress, so they could not given visit. Four of the data sets (for HAT-P-41b, WASP-74b, WASP-79b, and WASP-121b) only used forward scanning

WASP-121b occurred two years before the other four obserof $r_{1}$ and $r_{2}$ than the other four visits. noise ${ }^{34}$. constrain parameters such as the secondary eclipse time to the ${ }_{354}$ As described in the main text, we measured the dayside temlevel of precision provided by previous observations. There- ${ }_{355}$ perature of each observed planet by fitting a blackbody to the fore, all other eclipse parameters were fixed to the literature ${ }_{356}$ "out-of-band" regions indicated in Supplementary Figure 1. values listed in Supplementary Table 3. For the systematics ${ }_{357}$ Similar to previous studies $^{41}$, we found a linear relationship model, $c, v$, and $s$ were allowed to vary between orbits, while ${ }_{358}$ between this observed dayside temperature and the planetary $r_{1}$ and $r_{2}$ were fixed to the same values for all orbits within $\mathrm{a}_{359}$ irradiation temperature given by instead of bi-directional scanning, so for these observations ${ }_{360}$ where $T_{i r r}=T_{\text {eff }} \sqrt{R_{*} / a}$ is the irradiation temperature, $R_{*}$ is we fixed $s=1$. The first secondary eclipse observation for ${ }_{361}$ the stellar radius, and $a$ is the semi-major axis.

vations and showed significant differences in the ramp shape,з62 Model Grid

so we allowed this first eclispe to be fit with different values ${ }_{363}$ We created a new grid of self-consistent, 1D hot Jupiter

The data sets for WASP-76b and WASP-79b showed addi-365 tion of observed planets. These models were generated ustional correlated noise after applying this systematic model,366 ing the Sc-CHIMERA code (validated against established so for these two data sets we tested adding an additional 367 brown dwarf models ${ }^{42}$ and analytic models ${ }^{22}$ ) assuming cloudquadratic term to the visit-long trend. While adding this ad-368 free, radiative-convective-thermochemical equilibrium atmoditional term was able to correct for the correlated noise, it 369 spheres. The models' assumption of chemical equilibrium introduced strong degeneracies between the fit parameters and 370 is likely a good approximation for the highly irradiated planthe planet-to-star flux ratio. In order to avoid these degenera-371 ets that make up the majority of our observed population ${ }^{43}$. cies, we fit for only a linear visit-long trend in our final fit 372 A two stream source function technique ${ }^{44}$ is employed to and used the divide-white method to correct for the additional 373 solve for the planetary thermal fluxes at each atmospheric

We estimated the parameters with a Markov Chain Monte 375 (MCMC) fit using the emcee package for Python ${ }^{38} .376$ (for both direct and diffuse fluxes, under the quadrature apThe final secondary eclipse spectra for all of the planets are 377 proximation) assuming cosine incident angle of 0.5, utilizing 
378 the PHOENIX models for the stellar spectra ${ }^{45}$. A Newton-426

379 Raphson iteration ${ }^{46}$ is used to determine the temperature at ${ }_{427}$

380 each model layer which ensures zero net flux divergence. We 428

381 include absorption cross-sections from $0.1-100 \mu \mathrm{m}$ (where ${ }_{429}$

382 available) for $\mathrm{H}_{2} \mathrm{O}, \mathrm{CO}, \mathrm{CO}_{2}, \mathrm{CH}_{4}, \mathrm{NH}_{3}, \mathrm{H}_{2} \mathrm{~S}, \mathrm{PH}_{3}, \mathrm{HCN}, 430$ a

${ }_{383} \mathrm{C}_{2} \mathrm{H}_{2}$, TiO, VO, $\mathrm{SiO}, \mathrm{FeH}, \mathrm{CaH}, \mathrm{MgH}, \mathrm{CrH}, \mathrm{AlH}, \mathrm{Na}, \mathrm{K}, 431$

$384 \mathrm{Fe}, \mathrm{Mg}, \mathrm{Ca}, \mathrm{C}, \mathrm{Si}, \mathrm{Ti}, \mathrm{O}, \mathrm{Fe}^{+}, \mathrm{Mg}^{+}, \mathrm{Ti}^{+}, \mathrm{Ca}^{+}, \mathrm{C}^{+}, \mathrm{H}_{2,432}$ a

$385 \mathrm{H}_{2}-\mathrm{H}_{2} / \mathrm{He} \mathrm{CIA},{ }^{47-50}, \mathrm{H}^{-}$bound-free and free-free ${ }^{51,52}$, and ${ }_{433}$

$\mathrm{H}_{2} / \mathrm{He}$ Rayleigh scattering, and additional UV opacities for 434

$\mathrm{CO}, \mathrm{SiO}$, and $\mathrm{H}_{2}{ }^{49}$. Pre-computed cross-sections were con-435

verted into correlated-K coefficients at a spectral resolution 436

of 250 using a 10 point double Gauss quadrature (with half ${ }_{437} \mathrm{v}$

covering the top $5 \%$ of the correlated-K cumulative distribu-438

tion function) with mixed-gas optical depths computed using

the random-overlap resort-rebin framework (e.g., ${ }^{53,54}$ ). Ther-

mochemical equilibrium molecular abundances were com-439

puted using the NASA CEA Gibbs free energy minimization ${ }^{440}$

code $^{55}$ combined with elemental-rain out due to condensate ${ }^{441}$

formation (all major $\mathrm{Si}, \mathrm{Fe}, \mathrm{Mg}, \mathrm{Ca}, \mathrm{Al}, \mathrm{Na}$, and $\mathrm{K}$ bearing 442

condensates are included) given the Lodders et al. $(2009)^{56}{ }_{443}$

elemental abundances.

We parameterized the model atmospheres with a set of five ${ }^{445}$

399

400 parameters: the stellar effective temeprature $\left(T_{e f f}\right)$, the plane-446

401 tary gravity $(g)$, the planetary metallicity $\left(\left[\frac{\mathrm{M}}{\mathrm{H}}\right]\right)$, the planetary ${ }^{447}$

402 carbon-to-oxygen ratio $\left(\frac{\mathrm{C}}{\mathrm{O}}\right)$, and the planetary internal temper- ${ }^{448}$

403 ature $\left(T_{i n t}\right)$. Our fiducial models had the following parameter ${ }^{449}$

${ }_{404}$ values: $T_{e f f}=5300 \mathrm{~K}, g=10 \mathrm{~m} / \mathrm{s}^{2},\left[\frac{\mathrm{M}}{\mathrm{H}}\right]=0.0, \frac{\mathrm{C}}{\mathrm{O}}=0.55,{ }^{450}$

${ }_{405} T_{i n t}=150 \mathrm{~K}$. Models at different irradiation temperatures ${ }^{451}$

406 were created by re-scaling the incident stellar spectrum (the ${ }^{452}$

407 PHOENIX model for a given stellar effective temperature) ${ }^{453}$

408 by the ratio of the desired irradiation temperature to the bolo- ${ }^{454}$

409 metric temperature of a planet at 0.05 AU around a 1 solar

410 radius star. We created models with irradiation temperatures

411 between $500-3600 \mathrm{~K}$, with step sizes of $50-200 \mathrm{~K}$.

412 Following Lothringer \& Barman (2019) ${ }^{25}$, we calculate

${ }_{413}$ the absorption mean opacity $\kappa_{J}$ and the Planck mean opacity

${ }_{414} \kappa_{B}$ at a pressure of $10^{-2}$ bar as a function of equilibrium tem-

415 perature for our fiducial models. The absorption mean opacity

416 at a given pressure $P$ is given by

$$
\kappa_{J}(P)=\frac{\int_{0}^{\infty} \kappa_{\lambda}(T, P) J_{\lambda}(P) d \lambda}{\int_{0}^{\infty} J_{\lambda}(P) d \lambda},
$$

417 where $\kappa_{\lambda}$ is the monochromatic true absorption coefficient, ${ }_{418} J_{\lambda}$ is the mean intensity at a given wavelength, and $T$ is the ${ }_{419}$ local temperature in the planet's atmosphere ${ }^{25}$. The Planck ${ }_{467}$ 420 mean opacity is given by

$$
\kappa_{B}(P)=\frac{\int_{0}^{\infty} \kappa_{\lambda}(T, P) B_{\lambda}(T) d \lambda}{\int_{0}^{\infty} B_{\lambda}(T) d \lambda},
$$

421 where $B_{\lambda}(T)$ is the Planck function. The ratio $\kappa_{J} / \kappa_{B}$ de-472 scatter we see in water feature strengths and has no impact on 422 scribes the relative efficiency of stellar absorption vs. thermal 473 the feature strengths above $T_{\text {day }}=2000 \mathrm{~K}$. Our results agree 423 re-radiation, and a ratio $\kappa_{J} / \kappa_{B}>1$ generally indicates the 474 with those from general circulation models, which also show ${ }_{424}$ presence of a thermal inversion in the temperature-pressure 475 that clouds have little to no impact at temperatures above 425 profile.
We also created subset grids as a function of irradiation temperature where a single parameter dimension was varied while all other parameters were held fixed to their fiducial model values (no cross-variance). We examined models with stellar $T_{\text {eff }}=3300 \mathrm{~K}, 4300 \mathrm{~K}, 6300 \mathrm{~K}, 7200 \mathrm{~K}$, and $8200 \mathrm{~K}$; $g=1 \mathrm{~m} / \mathrm{s}^{2}$ and $100 \mathrm{~m} / \mathrm{s}^{2} ;\left[\frac{\mathrm{M}}{\mathrm{H}}\right]=-1.5$ and $1.5 ;$ and $\frac{\mathrm{C}}{\mathrm{O}}=0.01$ and 0.85 . For models with different metallicities, elemental bundance ratios were held constant while the overall metalicity was re-scaled relative to $\mathrm{H}$. We also created a model rid where the internal temperature varies with the planetary rradiation temperature following Equation 3 in $\operatorname{ref}\left({ }^{26}\right)$. Individual model tracks with irradiation temperature for each of hese variations are shown in Figure 4.

Opacity from gaseous $\mathrm{TiO} / \mathrm{VO}$ is theorized to be a driving force behind the transition between uninverted hot Jupiter atmospheres with monotonically decreasing temperaturepressure profiles and atmospheres containing thermal inversions $^{2}$. Some previous observations of hot Jupiters have suggested that vapor TiO/VO may not be present in hightemperature atmospheres if it is condensed in cooler parts of the atmosphere (e.g., ${ }^{28}$ ). This process, known as coldtrapping, effectively works to remove $\mathrm{TiO} / \mathrm{VO}$ from places in the atmosphere where vaporized $\mathrm{TiO} / \mathrm{VO}$ would be expected to be present in equilibrium. In order to study the impact of potential cold-trapping, we created models where the $\mathrm{TiO}$ and VO opacities are artificially set to zero until a given temperature threshold. We tested models where TiO/VO opacity is zeroed out for temperatures below $2000 \mathrm{~K}, 2500 \mathrm{~K}$, and $3000 \mathrm{~K}$. These tracks are also shown in Figure 4.

Recent studies have suggested clouds may have an impact on the strength of molecular absorption features observed in thermal emission (e.g., ${ }^{7,57}$ ).To test the impact the presence of clouds would have on the trends in our models, we created two cloudy models. We used the cloud model of Ackerman \& Marley $(2001)^{58}$, as implemented by Mai \& Line $(2019)^{59}$. Both 461 models had a constant vertical mixing strength of $10^{8} \mathrm{~cm}^{2} / \mathrm{s}$ 462 using the Zahnle et al. (2016) ${ }^{60}$ timescale prescription. We 463 tested models with sedimentation efficiencies of $f_{\text {sed }}=0.1$ 464 and 1.0. These models are shown compared to the fiducial model in Supplementary Figure 4. Adding clouds acts to weaken the water feature strengths below a dayside temperature of about $2000 \mathrm{~K}$, with a smaller $f_{\text {sed }}$ leading to more 468 effective weakening. While clouds may provide a potential ex469 planation for the weak water feature strength of HD 189733b, 6) 470 the lowest-temperature hot Jupiter in our population study,

471 we find that including clouds can not generally explain the $476 \approx 2000 \mathrm{~K}^{61,62}$. 


\section{${ }_{481}$ Correspondence and Requests}

${ }_{482}$ Correspondence and requests for materials should be addressed to M.M.

\section{${ }_{483}$ Acknowledgements}

${ }_{484}$ Based on observations made with the NASA/ESA Hubble Space Telescope, obtained from the data archive at the Space 485 Telescope Science Institute. STScI is operated by the Association of Universities for Research in Astronomy, Inc. under NASA contract NAS 5-26555. M.M. acknowledges funding from a NASA FINESST grant. M.R.L acknowledges funding from NSF AST-165220, and NASA NNX17AB56G. M.R.L also acknowledges opacity information from Roxana Lupu and Ehsan-Gharib 488 Nezhad. M.R.L., J.L.B., and J.J.F. acknowledge funding for this work from STScI grants GO-13467 and GO-14792. J.M.D 489 acknowledges support from the Amsterdam Academic Alliance (AAA) Program, and the European Research Council (ERC) 490 European Union's Horizon 2020 research and innovation programme (grant agreement no. 679633; Exo-Atmos). This work is 491 part of the research programme VIDI New Frontiers in Exoplanetary Climatology with project number 614.001.601, which is 492 (partly) financed by the Dutch Research Council (NWO).

\section{${ }_{493}$ Author contributions statement}

494 M.M. reduced and analyzed the new data sets, led the data-model comparison, and wrote the manuscript. M.R.L. created the 495 self-consistent 1D model grids and contributed to the writing of the manuscript. J.L.B. contributed to the conception of the

496 population study and the writing of the manuscript. J.J.F. contributed to the interpretation of the results and the writing of the 497 manuscript. V.P., E.M.-R.K., C.B., and J.-M.D. contributed to the interpretation of the results. D.K.S. and M.L.-M. are PIs of 498 the HST program GO-14767 from which we obtained the new observations that were analyzed in this work. M.R.S. and G.M.R 499 contributed to the conception of the population study. All authors commented on the manuscript.

500 Facilities: Hubble Space Telescope, Wide Field Camera 3

${ }_{501}$ Software: batman ${ }^{36}$, emcee $^{38}$, matplotlib ${ }^{63}$, numpy ${ }^{64}$, pysynphot $^{65}$, scipy ${ }^{66}$

\section{${ }_{502}$ Competing Interests}

The authors declare no competing financial interests.

\section{Supplementary Information References}

31. Edwards, B. et al. ARES I: WASP-76 b, A Tale of Two HST Spectra. AJ 160, 8 (2020). DOI 10.3847/1538-3881/ab9225. 2005.02374 .

32. Fu, G. et al. The Hubble PanCET program: Transit and Eclipse Spectroscopy of the Strongly Irradiated Giant Exoplanet WASP-76b. arXiv e-prints arXiv:2005.02568 (2020). 2005.02568.

33. Pluriel, W. et al. ARES. III. Unveiling the Two Faces of KELT-7 b with HST WFC3. AJ 160, 112 (2020). DOI 10.3847/1538-3881/aba000. 2006.14199.

34. Kreidberg, L. et al. Clouds in the atmosphere of the super-Earth exoplanet GJ1214b. Nat. 505, 69-72 (2014). DOI 10.1038/nature12888. 1401.0022 .

35. Horne, K. An optimal extraction algorithm for CCD spectroscopy. PASP 98, 609-617 (1986). DOI 10.1086/131801.

36. Kreidberg, L. batman: BAsic Transit Model cAlculatioN in Python. PASP 127, 1161 (2015). DOI 10.1086/683602. 1507.08285.

37. Berta, Z. K. et al. The Flat Transmission Spectrum of the Super-Earth GJ1214b from Wide Field Camera 3 on the Hubble Space Telescope. ApJ 747, 35 (2012). DOI 10.1088/0004-637X/747/1/35. 1111.5621.

38. Foreman-Mackey, D., Hogg, D. W., Lang, D. \& Goodman, J. emcee: The MCMC Hammer. PASP 125, 306 (2013). DOI 10.1086/670067. 1202.3665.

39. Castelli, F. \& Kurucz, R. L. New Grids of ATLAS9 Model Atmospheres. In Piskunov, N., Weiss, W. W. \& Gray, D. F. (eds.) Modelling of Stellar Atmospheres, vol. 210 of IAU Symposium, A20 (2003). astro-ph $/ 0405087$. 
40. Southworth, J., Bohn, A. J., Kenworthy, M. A., Ginski, C. \& Mancini, L. A multiplicity study of transiting exoplanet host stars. II. Revised properties of transiting planetary systems with companions. A\&A 635, A74 (2020). DOI 10.1051/00046361/201937334. 2001.08225.

41. Schwartz, J. C. \& Cowan, N. B. Balancing the energy budget of short-period giant planets: evidence for reflective clouds and optical absorbers. MNRAS 449, 4192-4203 (2015). DOI 10.1093/mnras/stv470. 1502.06970.

42. Saumon, D. \& Marley, M. S. The Evolution of L and T Dwarfs in Color-Magnitude Diagrams. ApJ 689, 1327-1344 (2008). DOI 10.1086/592734. 0808.2611.

43. Kitzmann, D. et al. The Peculiar Atmospheric Chemistry of KELT-9b. ApJ 863, 183 (2018). DOI 10.3847/15384357/aace5a. 1804.07137.

44. Toon, O. B., McKay, C. P., Ackerman, T. P. \& Santhanam, K. Rapid calculation of radiative heating rates and photodissociation rates in inhomogeneous multiple scattering atmospheres. JGR 94, 16287-16301 (1989). DOI 10.1029/JD094iD13p16287.

45. Husser, T. O. et al. A new extensive library of PHOENIX stellar atmospheres and synthetic spectra. A\&A 553, A6 (2013). DOI 10.1051/0004-6361/201219058. 1303.5632.

46. McKay, C. P., Pollack, J. B. \& Courtin, R. The thermal structure of Titan's atmosphere. Icarus 80, 23-53 (1989). DOI 10.1016/0019-1035(89)90160-7.

47. Lupu, R. E. et al. The Atmospheres of Earthlike Planets after Giant Impact Events. ApJ 784, 27 (2014). DOI 10.1088/0004637X/784/1/27. 1401.1499.

48. Tennyson, J. et al. The 2020 release of the ExoMol database: molecular line lists for exoplanet and other hot atmospheres. arXiv e-prints arXiv:2007.13022 (2020). 2007.13022.

49. Kurucz, R. \& Bell, B. Atomic Line Data. At. Line Data (R.L. Kurucz B. Bell) Kurucz CD-ROM No. 23. Camb. 23 (1995).

50. Gharib-Nezhad, E. \& Line, M. R. The Influence of $\mathrm{H}_{2} \mathrm{O}$ Pressure Broadening in High-metallicity Exoplanet Atmospheres. ApJ 872, 27 (2019). DOI 10.3847/1538-4357/aafb7b. 1809.02548.

51. Bell, K. L. \& Berrington, K. A. Free-free absorption coefficient of the negative hydrogen ion. J. Phys. B: At. 20, 1 (1987).

52. John, T. L. Continuous absorption by the negative hydrogen ion reconsidered. A\&A 193, 189-192 (1988).

53. Lacis, A. A. \& Oinas, V. A description of the correlated-k distribution method for modelling nongray gaseous absorption, thermal emission, and multiple scattering in vertically inhomogeneous atmospheres. JGR 96, 9027-9064 (1991). DOI 10.1029/90JD01945.

54. Amundsen, D. S. et al. The UK Met Office global circulation model with a sophisticated radiation scheme applied to the hot Jupiter HD 209458b. A\&A 595, A36 (2016). DOI 10.1051/0004-6361/201629183. 1608.08593.

55. Gordon, S. \& Mcbride, B. J. Computer program for calculation of complex chemical equilibrium compositions and applications. part 1: Analysis. Tech. Rep. 19950013764, NASA Lewis Research Center (1994).

56. Lodders, K., Palme, H. \& Gail, H. P. Abundances of the Elements in the Solar System. Landolt Börnstein 4B, 712 (2009). 0901.1149.

57. Taylor, J. et al. How Does Thermal Scattering Shape the Infrared Spectra of Cloudy Exoplanets? A Theoretical Framework and Consequences for Atmospheric Retrievals in the JWST era. arXiv e-prints arXiv:2009.12411 (2020). 2009.12411.

58. Ackerman, A. S. \& Marley, M. S. Precipitating Condensation Clouds in Substellar Atmospheres. ApJ 556, 872-884 (2001). DOI 10.1086/321540. astro-ph/0103423.

59. Mai, C. \& Line, M. R. Exploring Exoplanet Cloud Assumptions in JWST Transmission Spectra. ApJ 883, 144 (2019). DOI 10.3847/1538-4357/ab3e6d. 1908.10904.

60. Zahnle, K., Marley, M. S., Morley, C. V. \& Moses, J. I. Photolytic Hazes in the Atmosphere of 51 Eri b. ApJ 824, 137 (2016). DOI 10.3847/0004-637X/824/2/137. 1604.07388.

61. Roman, M. T. et al. Clouds in Three-Dimensional Models of Hot Jupiters Over a Wide Range of Temperatures I: Thermal Structures and Broadband Phase Curve Predictions. arXiv e-prints arXiv:2010.06936 (2020). 2010.06936.

62. Parmentier, V., Showman, A. P. \& Fortney, J. J. The cloudy shape of hot Jupiter thermal phase curves. arXiv e-prints arXiv:2010.06934 (2020). 2010.06934. 
63. Hunter, J. D. Matplotlib: A 2d graphics environment. Comput. In Sci. \& Eng. 9, 90-95 (2007). DOI 10.1109/MCSE.2007.55.

64. van der Walt, S., Colbert, S. C. \& Varoquaux, G. The NumPy Array: A Structure for Efficient Numerical Computation. Comput. Sci. Eng. 13, 22-30 (2011). DOI 10.1109/MCSE.2011.37. 1102 . 1523.

65. STScI Development Team. pysynphot: Synthetic photometry software package (2013). 1303.023.

66. Virtanen, P. et al. SciPy 1.0-Fundamental Algorithms for Scientific Computing in Python. arXiv e-prints arXiv:1907.10121 (2019). 1907.10121.

67. Bean, J. L. et al. Remastering the classics: A thermal inversion for the hot Jupiter archetype HAT-P-7b? HST Proposal (2016).

68. Sing, D. K. et al. The Panchromatic Comparative Exoplanetary Treasury Program. HST Proposal (2016).

69. Nikolov, N. et al. Hubble PanCET: an isothermal day-side atmosphere for the bloated gas-giant HAT-P-32Ab. MNRAS 474, 1705-1717 (2018). DOI 10.1093/mnras/stx2865. 1711.00859.

70. McCullough, P. Spanning the chasms: re-observing the transiting exoplanet HD 189733b. HST Proposal (2012).

71. Crouzet, N., McCullough, P. R., Deming, D. \& Madhusudhan, N. Water Vapor in the Spectrum of the Extrasolar Planet HD 189733b. II. The Eclipse. ApJ 795, 166 (2014). DOI 10.1088/0004-637X/795/2/166. 1409.4000.

72. Bean, J. Follow The Water: The Ultimate WFC3 Exoplanet Atmosphere Survey. HST Proposal (2013).

73. Line, M. R. et al. No Thermal Inversion and a Solar Water Abundance for the Hot Jupiter HD 209458b from HST/WFC3 Spectroscopy. AJ 152, 203 (2016). DOI 10.3847/0004-6256/152/6/203. 1605.08810.

74. Zhao, M. Near-IR spectroscopy of the newly discovered benchmark hot Jupiter WASP-103b. HST Proposal (2014).

75. Kreidberg, L. et al. Exploring the Frontier of Exoplanet Atmosphere Dynamics with NASA's Great Observatories. Spitzer Proposal (2014).

76. Evans, T. A global map of thermal inversions for an ultra-hot planet. HST Proposal (2017).

77. Stassun, K. G., Collins, K. A. \& Gaudi, B. S. Accurate Empirical Radii and Masses of Planets and Their Host Stars with Gaia Parallaxes. AJ 153, 136 (2017). DOI 10.3847/1538-3881/aa5df3. 1609.04389.

78. Johnson, M. C., Cochran, W. D., Addison, B. C., Tinney, C. G. \& Wright, D. J. Spin-Orbit Misalignments of Three Jovian Planets via Doppler Tomography. AJ 154, 137 (2017). DOI 10.3847/1538-3881/aa8462. 1708.01291.

79. Zhou, G. et al. Spin-orbit alignment for KELT-7b and HAT-P-56b via Doppler tomography with TRES. MNRAS 460, 3376-3383 (2016). DOI 10.1093/mnras/stw1107. 1605.01991.

80. Bonomo, A. S. et al. The GAPS Programme with HARPS-N at TNG . XIV. Investigating giant planet migration history via improved eccentricity and mass determination for 231 transiting planets. A\&A 602, A107 (2017). DOI 10.1051/00046361/201629882. 1704.00373.

81. West, R. G. et al. Three irradiated and bloated hot Jupiters:. WASP-76b, WASP-82b, and WASP-90b. A\&A 585, A126 (2016). DOI 10.1051/0004-6361/201527276. 1310.5607.

82. Delrez, L. et al. WASP-121 b: a hot Jupiter close to tidal disruption transiting an active F star. MNRAS 458, 4025-4043 (2016). DOI 10.1093/mnras/stw522. 1506.02471. 
Supplementary Tables

\begin{tabular}{cccc}
\hline Planet & HST Program \# & Number of Eclipses & Literature Reference \\
\hline \hline HAT-P-7b & $14792^{67}$ & 2 & Mansfield et al. $(2018)^{21}$ \\
HAT-P-32Ab & $14767^{68}$ & 1 & Nikolov et al. $(2018)^{69}$ \\
HD 189733b & $12881^{70}$ & 1 & Crouzet et al. $(2014)^{71}$ \\
HD 209458b & $13467^{72}$ & 5 & Line et al. $(2016)^{73}$ \\
WASP-18b & $13467^{72}$ & 5 & Arcangeli et al. $(2018)^{4}$ \\
WASP-43b & $13467^{72}$ & 5 & Kreidberg et al. $(2014)^{5}$ \\
WASP-103b & $13660^{74}, 14050^{75}$ & 4 & Kreidberg et al. $(2018)^{20}$ \\
\hline
\end{tabular}

Supplementary Table 1. Literature references and HST program numbers for the eight planets whose spectra were taken from the existing literature.

\begin{tabular}{cccccc}
\hline Planet & HST Program \# & $\begin{array}{c}\text { Date(s) of } \\
\text { Observation }\end{array}$ & Sampling Sequence & Exposure Time [s] & $\begin{array}{c}\text { Exposures } \\
\text { per Orbit }\end{array}$ \\
\hline \hline HAT-P-41b & $14767(1)^{68}$ & $10 / 09 / 16$ & SPARS10, NSAMP=12 & 81.089 & 19 \\
KELT-7b & $14767(1)^{68}$ & $08 / 18 / 17$ & SPARS10, NSAMP=4 & 22.317 & 37 \\
WASP-74b & $14767(1)^{68}$ & $05 / 02 / 17$ & SPARS25, NSAMP=4 & 69.617 & 19 \\
WASP-76b & $14767(1)^{68}$ & $11 / 03 / 16$ & SPARS10, NSAMP=15 & 103.129 & 19 \\
WASP-79b & $14767(1)^{68}$ & $11 / 15 / 16$ & SPARS25, NSAMP=7 & 138.381 & 13 \\
WASP-121b & $14767(1)^{68}$, & $11 / 10 / 16-11 / 11 / 16$, & SPARS10, NSAMP=15 & 103.129 & 16 \\
& $15134(4)^{76}$ & $03 / 12 / 18-03 / 13 / 18$, & & & \\
& \multicolumn{3}{c}{$03 / 14 / 18,02 / 03 / 19}$, \\
& $02 / 04 / 19$ & & & \\
\hline
\end{tabular}

Supplementary Table 2. Observing details for the six planets for which new data reductions were performed in this work. Numbers in parentheses next to the HST program number indicate the number of eclipses observed in that program. 


\begin{tabular}{cccccc}
\hline Planet & Period [days] & Mid-Transit Time [BJD] & $\mathrm{a} / \mathrm{r}_{*}$ & Inclination $\left[{ }^{\circ}\right]$ & $\mathrm{r}_{\mathrm{p}} / \mathrm{r}_{*}$ \\
\hline \hline HAT-P-41b & $2.694050^{77}$ & $2454983.8617^{78}$ & $5.45^{77}$ & $87.70^{77}$ & $0.1028^{78}$ \\
KELT-7b & $2.734770^{77}$ & $2456355.2293^{79}$ & $5.50^{77}$ & $83.76^{77}$ & $0.0888^{33}$ \\
WASP-74b & $2.137750^{77}$ & $2456506.8926^{80}$ & $4.86^{77}$ & $79.81^{77}$ & $0.0980^{77}$ \\
WASP-76b & $1.809882^{32}$ & $2456107.8551^{81}$ & $4.08^{32}$ & $88.50^{32}$ & $0.1087^{32}$ \\
WASP-79b & $3.662380^{77}$ & $2455545.2361^{80}$ & $7.03^{77}$ & $85.40^{77}$ & $0.1049^{77}$ \\
WASP-121b & $1.274926^{82}$ & $2456635.7083^{82}$ & $3.75^{82}$ & $87.60^{82}$ & $0.1245^{82}$ \\
\hline
\end{tabular}

Supplementary Table 3. Literature values for fixed eclipse parameters in the light curve models for the six data sets reduced in this work.

\begin{tabular}{c|cccccc}
\hline Wavelength $[\mu \mathrm{m}]$ & HAT-P-41b & KELT-7b & WASP-74b & WASP-76b & WASP-79b & WASP-121b \\
\hline \hline $1.120-1.159$ & $207 \pm 157$ & $284 \pm 51$ & $288 \pm 67$ & $424 \pm 44$ & $12 \pm 33$ & $914 \pm 32$ \\
$1.159-1.197$ & $461 \pm 140$ & $328 \pm 45$ & $357 \pm 54$ & $589 \pm 33$ & $58 \pm 60$ & $956 \pm 32$ \\
$1.197-1.236$ & $622 \pm 91$ & $328 \pm 49$ & $304 \pm 57$ & $614 \pm 37$ & $297 \pm 54$ & $1009 \pm 33$ \\
$1.236-1.274$ & $545 \pm 95$ & $318 \pm 54$ & $310 \pm 55$ & $533 \pm 35$ & $298 \pm 64$ & $1001 \pm 29$ \\
$1.274-1.313$ & $452 \pm 84$ & $368 \pm 48$ & $429 \pm 48$ & $645 \pm 35$ & $155 \pm 49$ & $996 \pm 30$ \\
$1.313-1.351$ & $503 \pm 79$ & $321 \pm 50$ & $401 \pm 50$ & $723 \pm 33$ & $272 \pm 54$ & $1079 \pm 32$ \\
$1.351-1.390$ & $590 \pm 81$ & $371 \pm 54$ & $407 \pm 67$ & $804 \pm 33$ & $92 \pm 50$ & $1206 \pm 30$ \\
$1.390-1.429$ & $515 \pm 82$ & $415 \pm 51$ & $346 \pm 50$ & $739 \pm 36$ & $186 \pm 52$ & $1309 \pm 31$ \\
$1.429-1.467$ & $561 \pm 84$ & $411 \pm 53$ & $486 \pm 59$ & $980 \pm 37$ & $116 \pm 53$ & $1266 \pm 31$ \\
$1.467-1.506$ & $501 \pm 87$ & $445 \pm 55$ & $428 \pm 51$ & $1027 \pm 35$ & $130 \pm 58$ & $1362 \pm 32$ \\
$1.506-1.544$ & $666 \pm 89$ & $424 \pm 56$ & $428 \pm 53$ & $993 \pm 37$ & $242 \pm 57$ & $1311 \pm 36$ \\
$1.544-1.583$ & $613 \pm 96$ & $439 \pm 56$ & $560 \pm 56$ & $1273 \pm 40$ & $185 \pm 59$ & $1370 \pm 36$ \\
$1.583-1.621$ & $687 \pm 96$ & $447 \pm 64$ & $633 \pm 71$ & $970 \pm 45$ & $333 \pm 70$ & $1352 \pm 39$ \\
$1.621-1.660$ & $733 \pm 106$ & $392 \pm 61$ & $527 \pm 61$ & $909 \pm 47$ & $168 \pm 72$ & $1322 \pm 40$ \\
\hline
\end{tabular}

Supplementary Table 4. Secondary eclipse spectra for the six planets for which new data reductions were performed in this work. All eclipse depths are in units of ppm. 


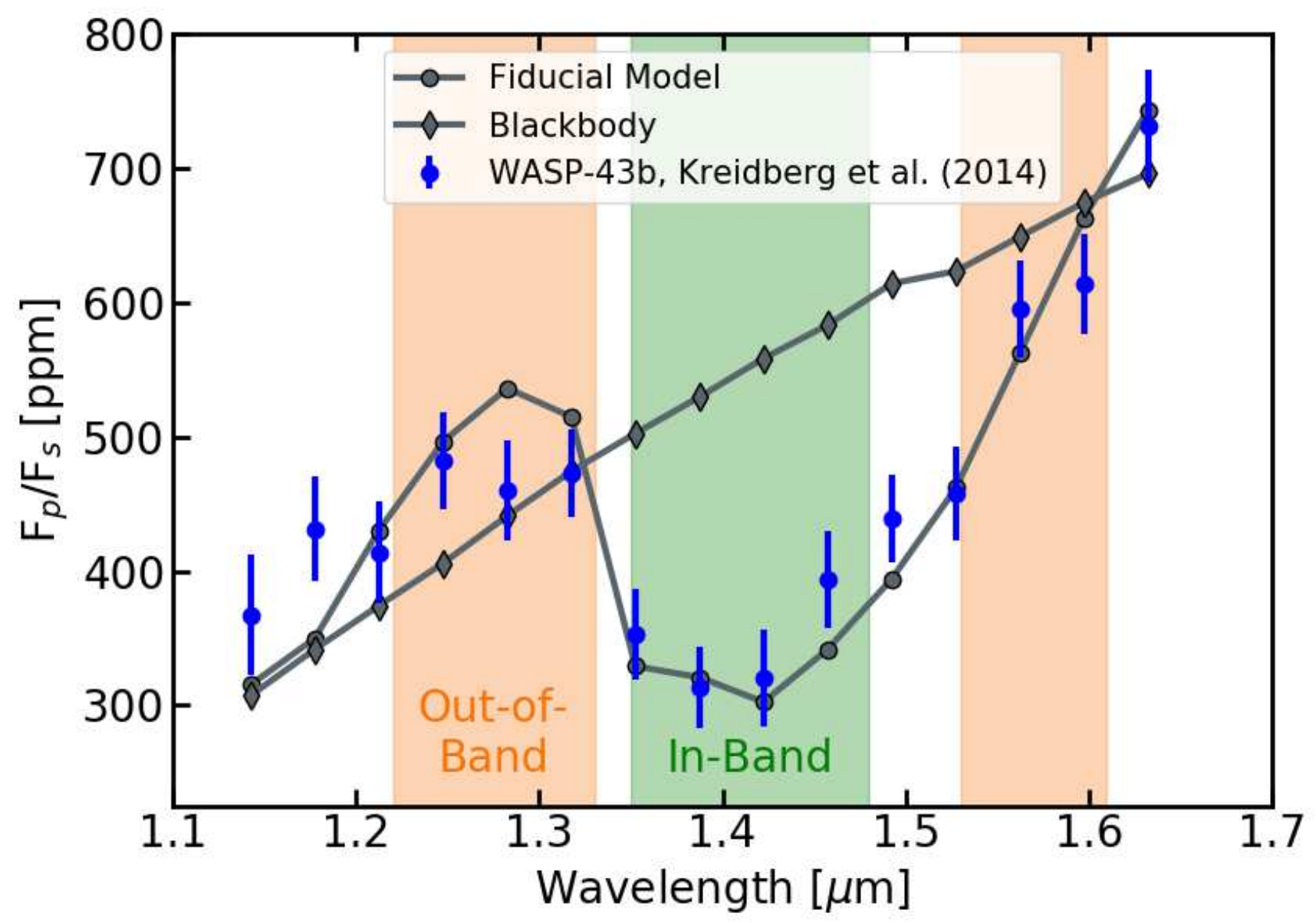

Supplementary Figure 1. Construction of the HST water feature strength metric to compare observed spectra to models. Blue points show $H S T /$ WFC 3 observations of WASP- $43 \mathrm{~b}^{5}$. The orange and green shaded regions indicate the spectral extent of the "out-of-band" and "in-band" flux, which are defined based on where the models in Figure 2 show water features. The gray line with circular points shows the best-fit model interpolated from those in Figure 2. The gray line with diamond-shaped points shows a blackbody fit to the out-of-band flux region. 


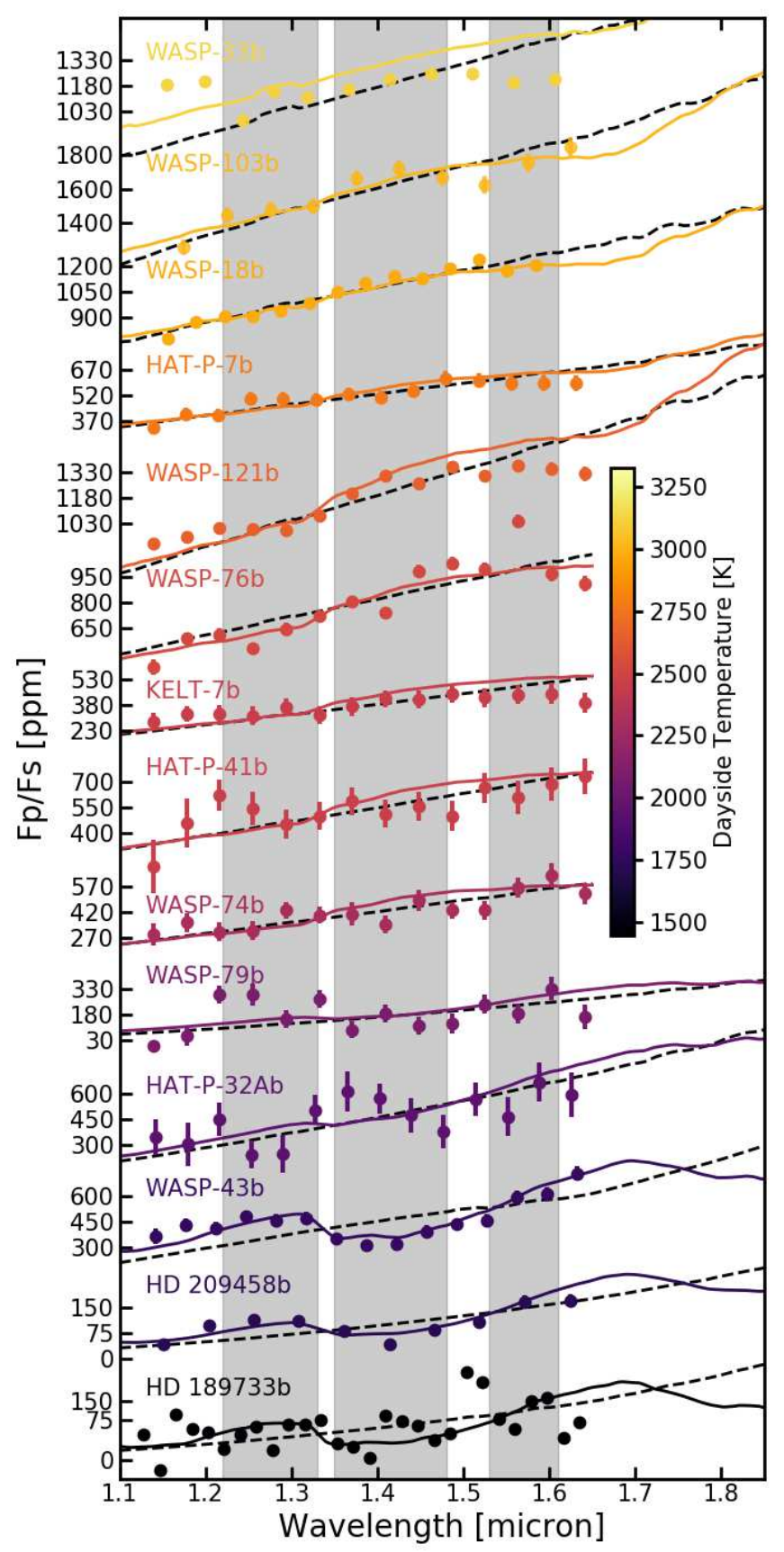

Supplementary Figure 2. Same as Figure 1, but with shaded bands indicating the "out-of-band" and "in-band" regions used to calculate the water feature strength $\left(S_{H_{2}} \mathrm{O}\right)$ for each observed secondary eclipse spectrum. 


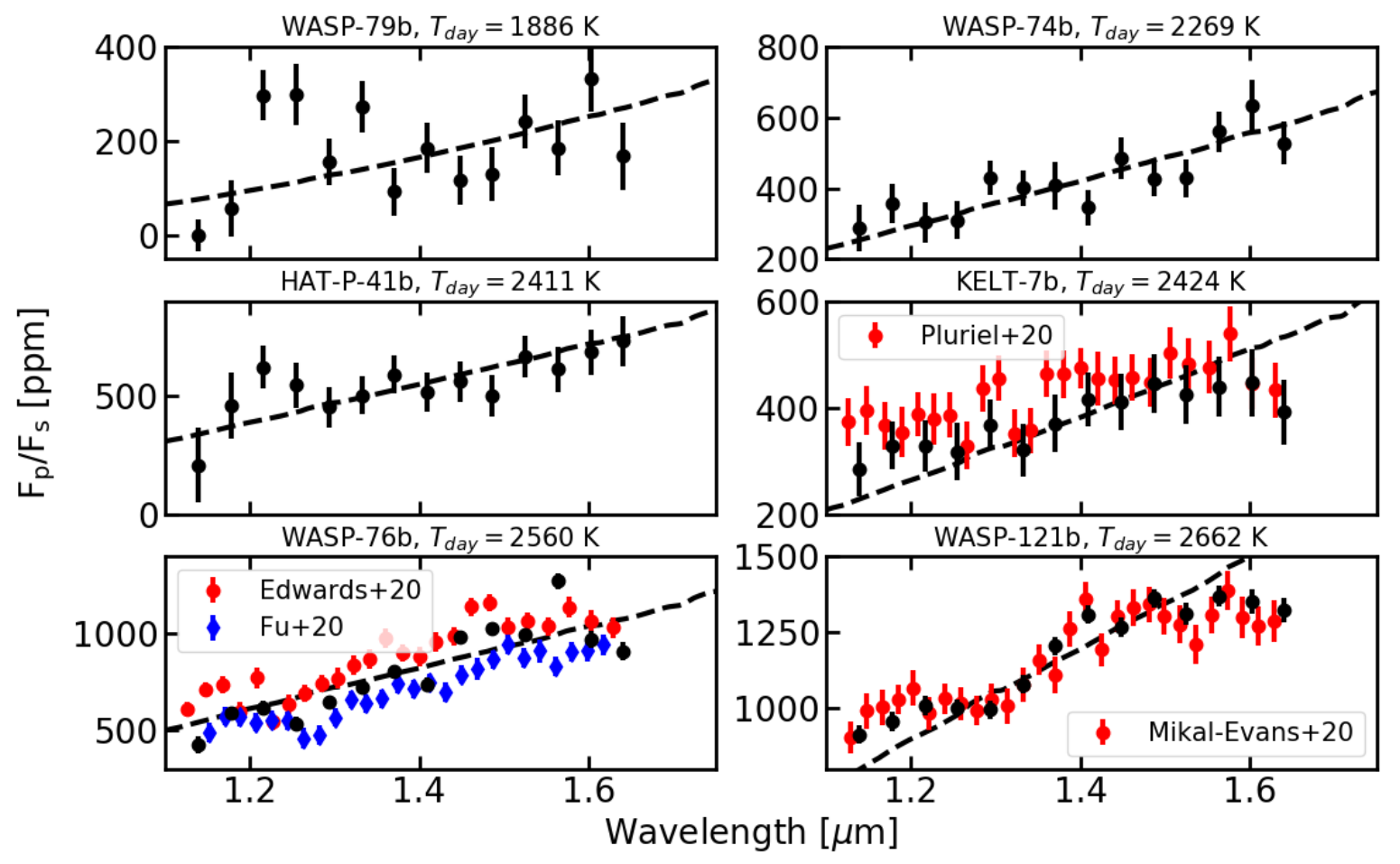

Supplementary Figure 3. HST/WFC3 secondary eclipse spectra for the six data reductions presented in this paper (black points). Black dashed lines indicate best-fit blackbody spectra, and temperatures above each plot give the corresponding dayside temperature $T_{d a y}$. Red points show previous data reductions from the literature ${ }^{6,31-33}$, which all show good agreement with the results presented here. 


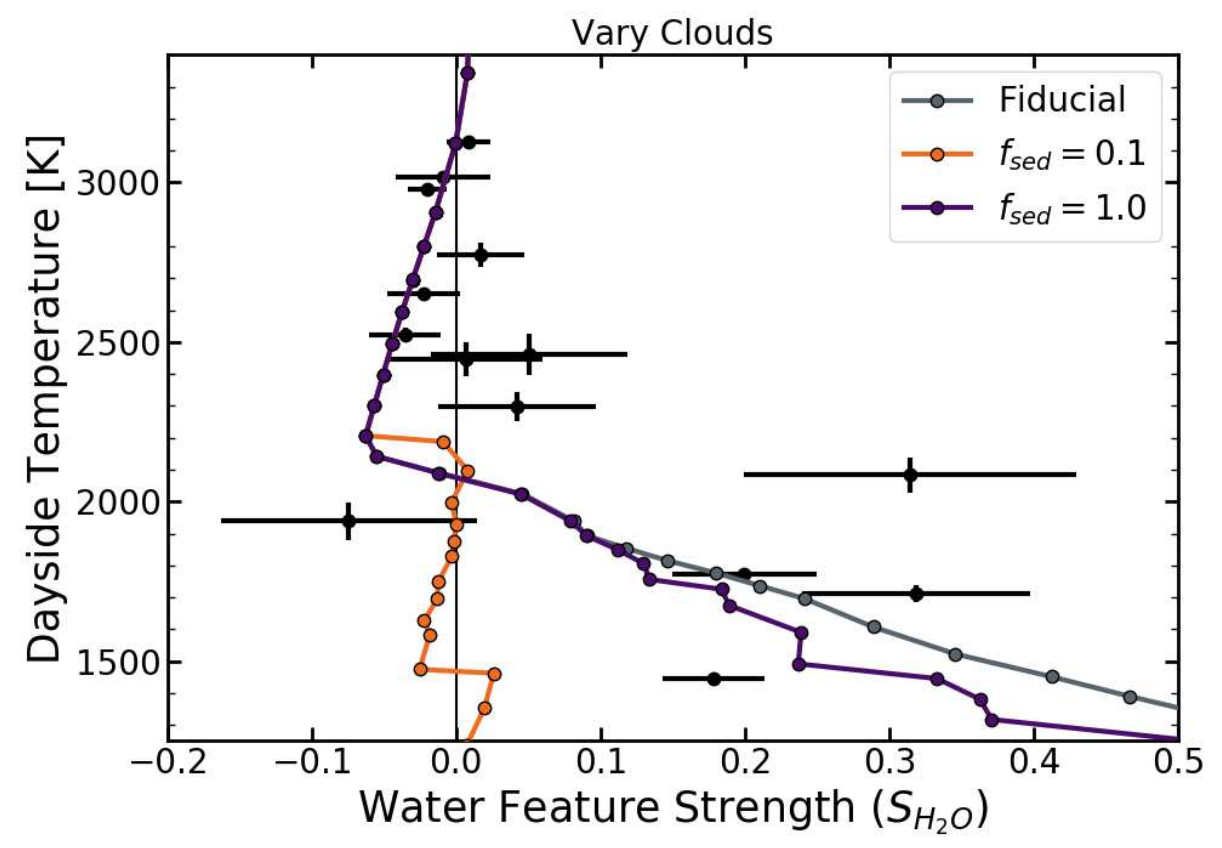

Supplementary Figure 4. Change in $H S T$ water feature strength when clouds are added to the fiducial model. The grey line shows the fiducial model, while the orange and purple lines show cloudy models with sedimentation efficiencies of $f_{\text {sed }}=0.1$ and 1.0, respectively. Adding clouds to the model effectively weakens the water feature strengths and makes the emission spectra more blackbody-like below dayside temperatures of about $2000 \mathrm{~K}$. However, clouds have no effect on $\mathrm{S}_{\mathrm{H}_{2} \mathrm{O}}$ at $T_{d a y} \geq 2000 \mathrm{~K}$ because the planets' daysides are too hot for any condensation to occur. 
Figures

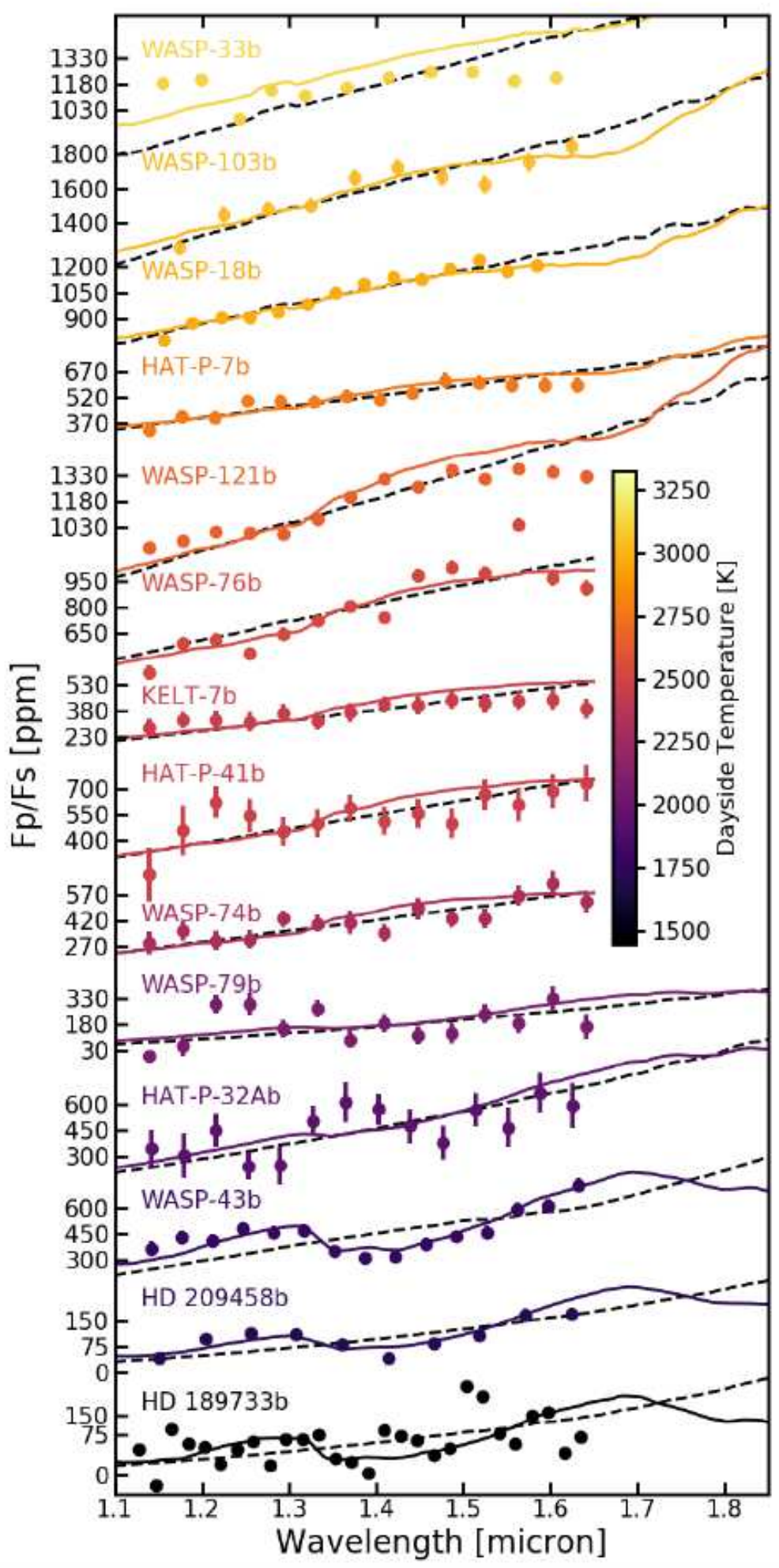

Figure 1

Secondary eclipse spectra for all 14 hot Jupiters considered in this study. Data sets are colored by dayside temperature, which is measured as described in the Methods and shown by the colorbar. Solid lines indicate interpolations from our solar composition fiducial model grid (see the Methods section for a 
description), while dashed lines indicate best-fit blackbodies. Note that for several data sets, the error bars are smaller than the point size.
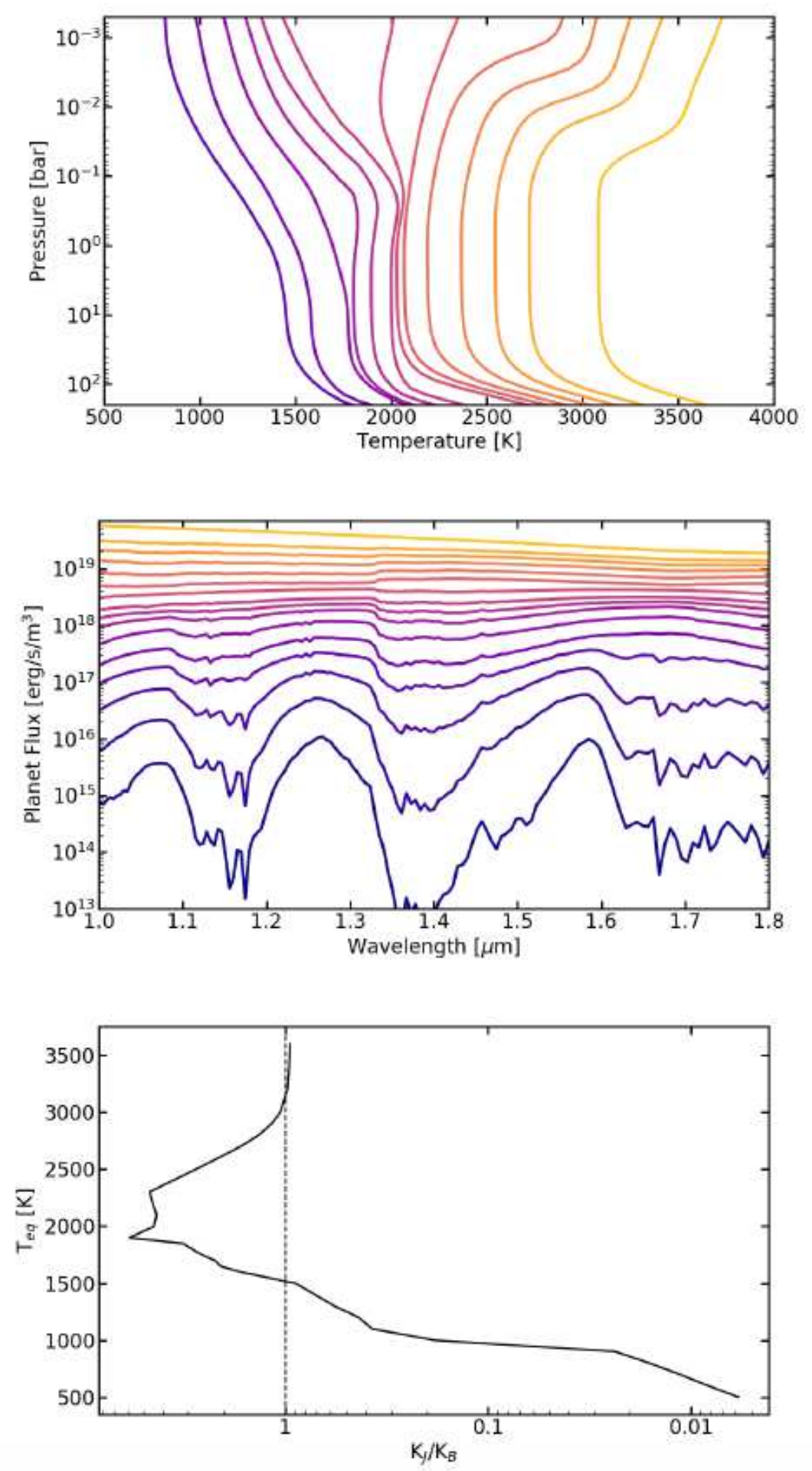

\section{Figure 2}

Temperature-pressure (T-P) profiles (top) and resulting dayside planet fluxes (middle) for the fiducial model grid, which covers approximately the same range of temperatures as spanned by the observations. The full model specifications are detailed in the Methods. The fiducial model uses a $5300 \mathrm{~K}$ stellar 
effective temperature, a solar composition planetary atmosphere $([\mathrm{M} / \mathrm{H}]=0.0$ and $\mathrm{C} / \mathrm{O}=0.55)$, a planetary gravity of $10 \mathrm{~m} / \mathrm{s} 2$, and a planet internal temperature of $150 \mathrm{~K}$. Blue and yellow lines show models with the coolest and warmest irradiation temperatures, respectively. For clarity, only every other model in the grid is shown here. The bottom panel shows the ratio of the absorption mean opacity (kJ) to the Planck mean opacity $(\mathrm{kB})$ as a function of equilibrium temperature in these models at a pressure of $10^{\wedge}-2$ bar. This ratio describes the relative efficiency of heating vs. cooling in the models 25 , and a ratio of $\mathrm{kJ} / \mathrm{kB}>1$ generally indicates the presence of a thermal inversion in the temperature-pressure profile. This panel is plotted with temperature on the $y$-axis for ease of comparison to Figure 3.

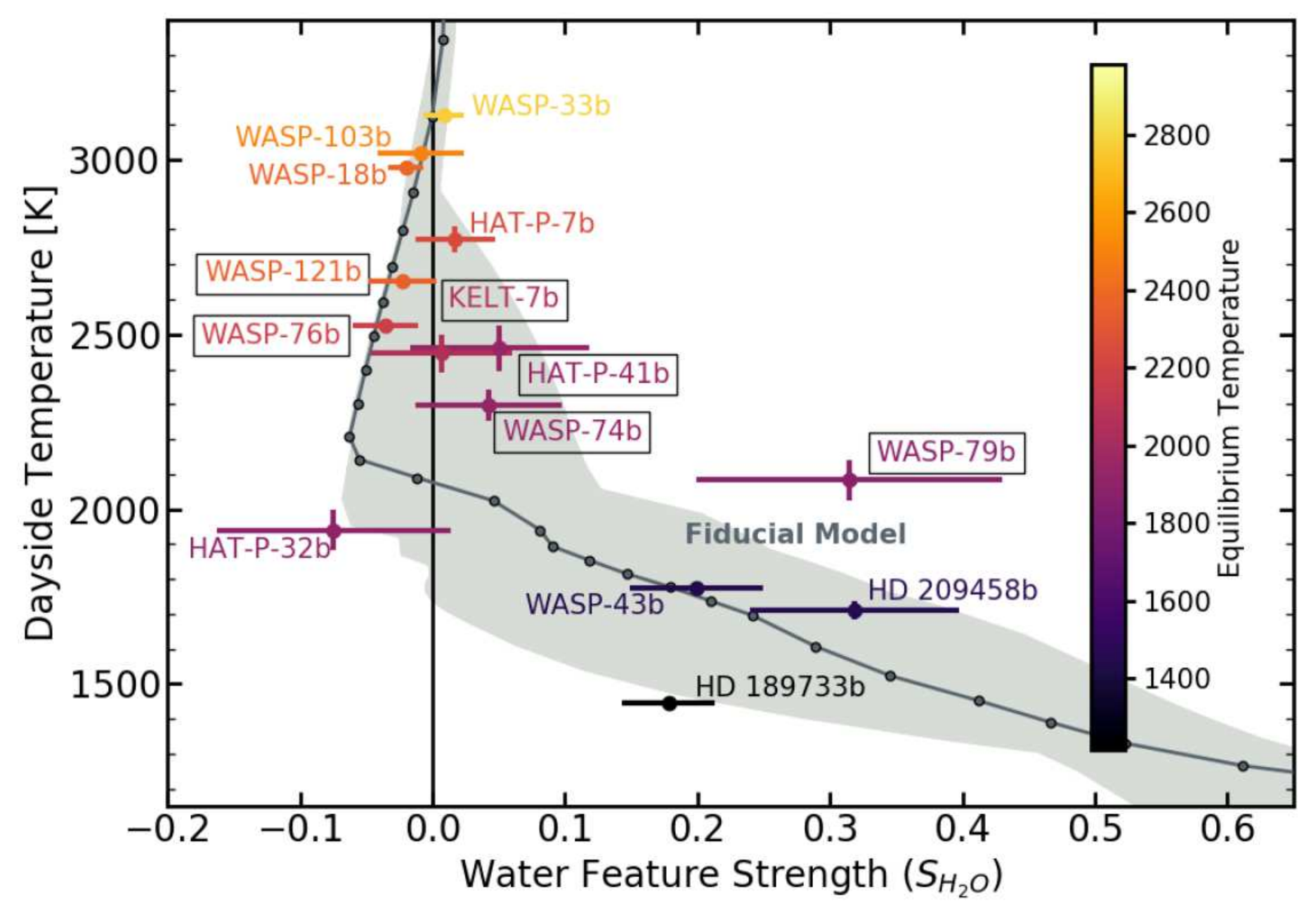

\section{Figure 3}

HST water feature strength diagram comparing observed secondary eclipse spectra to the model predictions in Figure 2. The y-axis shows the temperature of a blackbody fit to the "out-of-band" regions defined in Supplementary Figure 1, which is the observed dayside temperature Tday. The x-axis shows the strength of the observed feature in the water band at $1.4 \mu \mathrm{m}$ compared to this blackbody, as defined by Equation 1. Featureless, blackbody-like spectra have $\mathrm{SH} 2 \mathrm{O}=0$ and absorption/emission features have positive/negative colors, respectively. The gray line and points show the fiducial models pictured in Figure 2. The light gray shaded region shows the full range of model predictions assuming different values for the stellar effective temperature; the temperature where TiO opacity becomes important; and the planet 
gravity, C/O ratio, metallicity, and internal heat. Colored points with error bars show all planets with HST/WFC3 spectra observed in the spatial scanning mode, and boxes around planet names indicate new data reductions in this publication. The color scale indicates the planetary equilibrium temperature. The error bars include uncertainties in the stellar effective temperature.
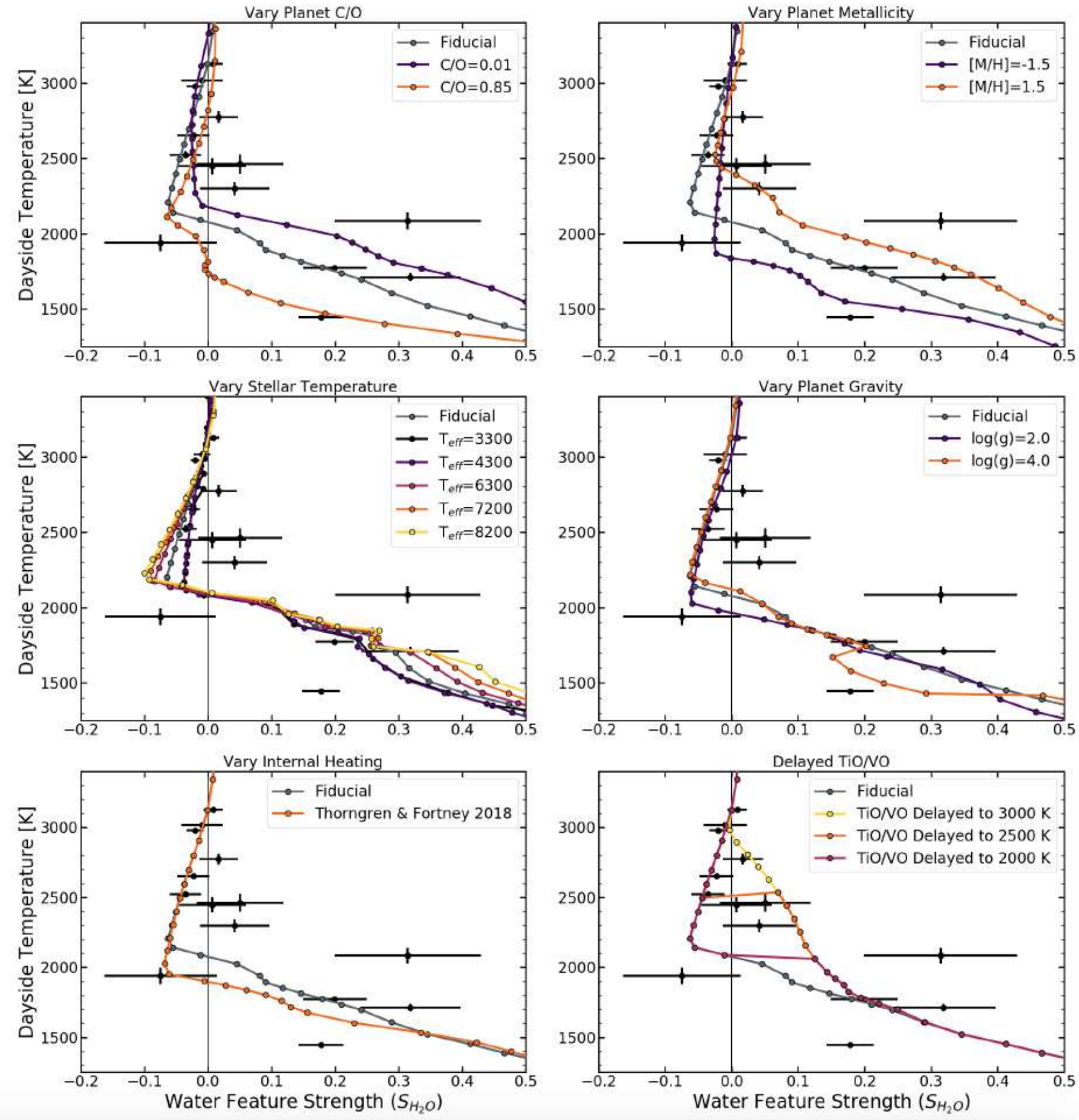

\section{Figure 4}

Diagrams illustrating the change in HST water feature strength from models with different parameters. All diagrams show the observed data as black points with error bars, while the lines show tracks for models with varying $\mathrm{C} / \mathrm{O}$ ratio (top left), metallicity (top right), stellar temperature (middle left), gravity 
(middle right), internal heating (bottom left), and the temperature to which TiO opacity was ignored (bottom right). In each case all other parameters are held fixed at the fiducial model values. The error bars include uncertainties in the stellar effective temperature. We found that changing the stellar temperature, planetary gravity, and internal heating in our models had little impact on the derived water feature strengths, and changing the TiO/VO only had an impact at intermediate temperatures, but changing the atmospheric $\mathrm{C} / \mathrm{O}$ ratio and metallicity can explain the diversity of observed secondary eclipse spectra. 